\title{
SOME NEW ANALYTICAL TECHNIQUES AND THEIR APPLICATION TO IRREGULAR CASES FOR THE THIRD ORDER ORDINARY LINEAR BOUNDARY-VALUE PROBLEM( $\left.{ }^{(}\right)$
}

\author{
BY
}

NATHANIEL R. STANLEY( $(2)$

\section{Abstract}

1. For the operator $T_{3}^{-}(D)$ defined by $-d^{3} / d x^{3}$ and a triple of boundary conditions irregular in the sense of Birkhoff, the reduction of this triple to canonical forms is implicit in the reduction made for a more general third order operator (Theorem 1.2).

2. A new technique is developed for calculating the Green's function for the $n$th order ordinary linear boundary-value problem (Theorem 2.4), and is applied to $T_{\overline{3}}^{-}$; a necessary and sufficient condition is given for the identification of degenerate sets of boundary conditions for $T_{3}^{-}$(Theorem 2.6).

3. A new technique is developed for calculating asymptotic expansions for large zeros of exponential sums, and the form of the expansion, which includes a logarithmic asymptotic series, is established by induction (Theorem 3.1); expansions for the cube roots of the eigenvalues of $T_{3}^{-}$then follow as special cases.

4. A theorem of Dunford and Schwartz (Theorem 4.0) giving a sufficient condition for completeness of eigenfunctions in terms of growth of the norm of the resolvent operator, is applied to prove that, with a possible exception, the eigenfunctions of $T_{\overline{3}}^{-}$span $L_{2}(0,1)$ (Theorem 4.5).

0 . Introduction. Some of the subject matter to be treated relates directly to the $n$th order ordinary linear differential operator $T_{n}(D)$ defined by

$$
L(D) y \equiv p_{0}(x) D^{n} y+p_{1}(x) D^{n-1} y+\cdots+p_{n}(x) y,
$$

in which the coefficient functions $p_{j}(x)(j=0,1, \cdots, n)$ of the real variable $x$ are continuous and $p_{0}(x) \neq 0$ in the domain of $y$, and by the set of $n$ linearly

Presented to the Society, October 31, 1959 under the titles Asymptotic expansions for large zeros of a class of exponential sums and $A$ simplified calculation of the Green's function for the ordinary linear boundary value problem; received by the editors January 30, 1961.

(1) In addition to the subject matter in the author's Ph.D. thesis, Irregular cases for the third order ordinary linear boundary-value problem, New York University 1959, the present paper contains generalizations of the Green's function method and of the analysis of exponential sums respectively. The author is indebted to his thesis adviser, Professor J. T. Schwartz.

(2) Adjunct Assistant Professor of Mathematics, New York University, and Manager, Applied Mathematics Department, Remington Rand Univac Division of Sperry Rand Corporation. 
independent homogeneous two-point boundary conditions

$$
\sum_{i=1}^{n}\left[a_{i, n-\imath} y^{(n-\imath)}(0)+b_{i, n-\imath} y^{(n-\imath)}(1)\right]=0 \quad(i=1, \cdots, n),
$$

with the coefficients $a_{i, n \rightarrow}$ and $b_{i, n \rightarrow}$ complex. The operator $T_{s}^{-}(D)$, defined by $L(D)=-D^{8}$ and BC's (0.2) (for $n=3$ ) irregular in the sense of Birkhoff, will be treated in detail. The following definitions and corresponding abbreviations (indicated in square brackets) are made:

A boundary form [BF, $U_{i}(y)$ ] is the left-hand member of a linear boundary condition $[\mathrm{BC}]$ given by $(0.2)$.

The boundary matrix [BM] is the matrix of coefficients $a_{i, n-a}, b_{i, n-\varepsilon}$ of the BF's (0.2), with not necessarily the same ordering of columns as in (0.2).

The order $\left[k_{i}\right]$ of a $\mathrm{BF}$ is the order of the derivative of highest order in it with a nonzero coefficient.

A normalized set of $n$ linearly independent [LI] BC's has at most two BF's of order $n-1, n-2, \cdots, 1$ respectively, arranged so that $k_{1} \geqq k_{2} \geqq \cdots$ $\geqq k_{n-1} \geqq k_{n} \geqq 0$, and $k_{i}>k_{i+2}$. Any set of $n$ LI BC's can be normalized by means of linear combinations [LC's] of the BF's.

Equivalent sets of $B C$ 's are such that each $\mathrm{BF}$ of one set is expressible as a LC of the BF's of the other set.

$\mathrm{BC}$ 's regular in the Birkhoff sense $[\mathrm{R}(\mathrm{B})]$ are such that for odd $n=2 m-1$, $\theta_{0}$ and $\theta_{1}$ are both not zero, and for even $n=2 m, \theta_{-1}, \theta_{1}$ are both not zero in the following identities, in which $a_{i} \equiv a_{i, k_{i}}, b_{i} \equiv b_{i, k_{i}}$ :

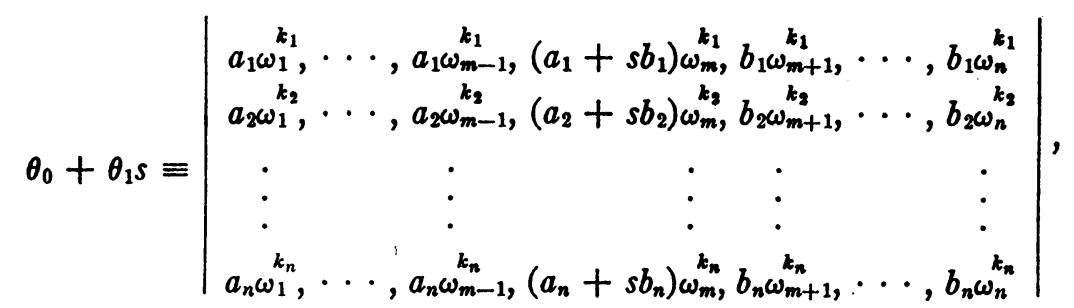

$$
\begin{aligned}
& s^{-1} \theta_{-1}+\theta_{0}+\theta_{1} s
\end{aligned}
$$

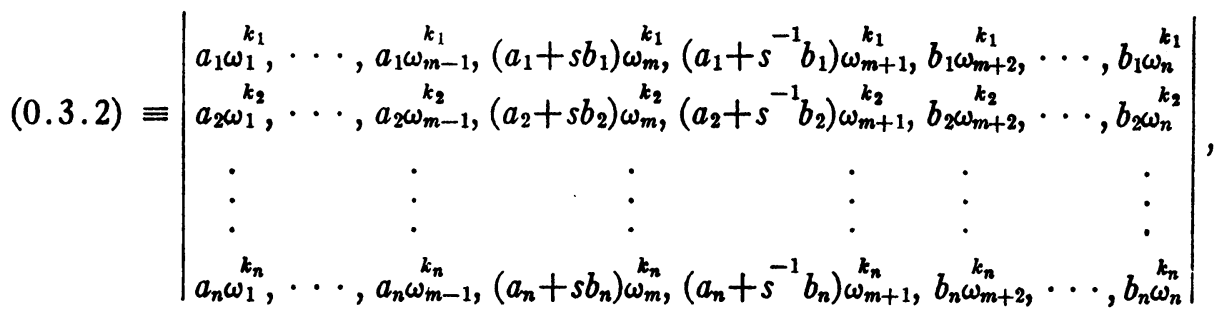

where $\omega_{1}, \cdots, \omega_{n}$ are the $n$th roots of -1 ordered so that $\operatorname{Re}\left(\rho \omega_{1}\right) \leqq \cdots$ $\leqq \operatorname{Re}\left(\rho \omega_{n}\right)$, and $\rho$ is some complex number. BC's irregular in the Birkhoff sense [I(B)] are such that $\theta_{0} \theta_{1}=0$ for odd $n=2 m-1$, and $\theta_{-1} \theta_{1}=0$ for even $n=2 m$. 
A now classical paper on the expansion of functions in an infinite series whose terms are eigenfunctions of an ordinary linear differential operator defined by BC's $R(B)$ was written by Birkhoff [2]. Subsequently, the corresponding problems for certain second or third order differential operators defined by irregular boundary conditions were treated by Hopkins [8], Stone [14], and Ward [16]. In [14], the series obtained was summable by means, rather than convergent in the usual sense; in [8] and [14], special irregular $\mathrm{BC}$ 's were considered, and strong restrictions were placed on the type of function to be expanded. Hoffman [7] has proved that the operators $\pm d^{2} / d x^{2}$ on $L_{2}(0,1)$ (or those differing from them by bounded perturbations) associated with BC's I.(B), have complete sets of eigenfunctions, but are not spectral. In $\S 4$ it will be proved that $T_{3}^{-}(D)$ has a complete set of eigenfunctions under the condition stated there.

1. Classification of third order boundary conditions. Each I(B) set of BC's for the third order operator $T_{3}(D)$, which includes $T_{3}^{-}$as a special case, will now be reduced to one of fourteen canonical sets of BC's, thereby simplifying identification of $I(B)$ sets of BC's and calculation with them. The $\mathrm{BM}$ will be used to designate the BC's. It is assumed that the BC's are normalized. The following table then gives all possible orders $k_{i}(i=1,2,3)$ for the BF's:

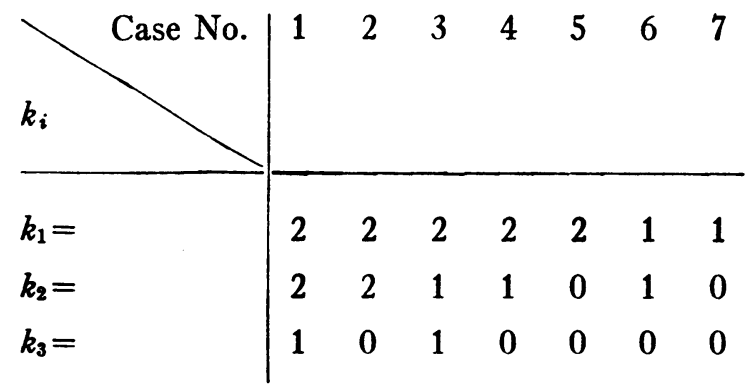

Since $k_{3}<2$, we have $a_{32}=b_{32}=0$. Since $k_{2}<2$ in Cases $3-7$, we have $a_{22}=b_{22}=0$ in these cases. In Cases 1 and 2, it is permissible to take one, but not both, of $a_{22}$ and $b_{22}$ as 0 , for the reasons which follow. Let $k_{i}=k_{i+1}$. The submatrix

$$
\left(\begin{array}{ll}
a_{i} & b_{i} \\
a_{i+1} & b_{i+1}
\end{array}\right)
$$

of the BM cannot have a null row, by the definition of order of a BF. If it has a null column, then the order of one of the BF's can be reduced by means of a LC, thus resulting in an equivalent pair of BC's and a later case (in Cases 5 and 7, the LI BF's preclude a null column). If both elements of a column are not zero, then a suitable LC will replace one of them by zero. Consequently, both elements of one diagonal or the other can be replaced by 
0's, thus giving two (mutually) symmetric subcases. These replacements will be made shortly in each of the six cases to which they apply.

Upon listing the conditions on the elements of the $B M$ in the seven respective cases, and choosing $\omega_{l}=e^{i(2 l-1) \pi / 3}(l=1,2,3), \rho=i$, where $i \equiv(-1)^{1 / 2}$, expanding the determinant in (0.3.1) leads to further conditions on these elements in the $R(B)$ and $I(B)$ (abbreviated as $R$ and $I$ ) subcases, as indicated below. All cases except Case 4 involve two subcases which are symmetric in the sense indicated above. Only one of the two has been selected for each such case; selection of the other merely results in the further conditions (labelled $\mathrm{R}$ or I) with the letters $a$ and $b$ interchanged. For brevity, the subcase has been indicated by specifying only one of the nonzero elements. In Case 1, for example, the statement $b_{22} \neq 0$ implies $a_{12} \neq 0$, and $a_{22}=b_{12}=0$. The further conditions, which result from either $a_{i}=0$ or $a_{i+1}=0$ and $\theta_{0}=0$ or $\theta_{1}=0$, are rejected when inconsistent with the particular subcase treated. In Case 1, for example, the conditions including $a_{12}=0$ are rejected as inconsistent in this sense.

CASE 1. $\left|a_{12}\right|+\left|b_{12}\right|>0, b_{22} \neq 0,\left|a_{31}\right|+\left|b_{31}\right|>0$.

$\mathrm{R}: a_{12} a_{31} b_{31} \neq 0$,

I: $a_{12}=0, b_{12} \neq 0$,

I: $a_{12} \neq 0, a_{81}=0, b_{81} \neq 0$,

I: $a_{12} \neq 0, a_{31} \neq 0, b_{31}=0$.

C.ASE 2. $\left|a_{12}\right|+\left|b_{12}\right|>0, b_{22} \neq 0,\left|a_{30}\right|+\left|b_{30}\right|>0$.

$$
\begin{aligned}
& \text { R: } a_{12} a_{30} b_{30} \neq 0, \\
& \text { I: } a_{12}=0, b_{12} \neq 0, \\
& \text { I: } a_{12} \neq 0, a_{30}=0, b_{30} \neq 0, \\
& \text { I: } a_{12} \neq 0, a_{30} \neq 0, b_{30}=0 .
\end{aligned}
$$

CASE 3. $\left|a_{12}\right|+\left|b_{12}\right|>0, b_{21} \neq 0,\left|a_{31}\right|+\left|b_{31}\right|>0$.

$$
\begin{aligned}
& \text { R: } a_{12} b_{12} a_{31} \neq 0, \\
& \text { I: } a_{31}=0, b_{31} \neq 0, \\
& \text { I: } a_{31} \neq 0, a_{12}=0, b_{12} \neq 0, \\
& \text { I: } a_{31} \neq 0, a_{12} \neq 0, b_{12}=0 .
\end{aligned}
$$

CASE 4. $\left|a_{12}\right|+\left|b_{12}\right|>0,\left|a_{21}\right|+\left|b_{21}\right|>0,\left|a_{30}\right|+\left|b_{30}\right|>0$.

$$
\mathrm{R}: \frac{\theta_{0}}{-(-3)^{1 / 2}} \equiv a_{12} a_{21} b_{30}+a_{12} b_{21} a_{30}+b_{12} a_{21} a_{30} \neq 0
$$




$$
\frac{\theta_{1}}{-(-3)^{1 / 2}} \equiv b_{12} b_{21} a_{30}+b_{12} a_{21} b_{30}+a_{12} b_{21} b_{30} \neq 0 \text {. }
$$

I: Only one of the above expressions equals zero.

I: Each of the above expressions equals zero.

CASE 5. $\left|a_{12}\right|+\left|b_{12}\right|>0, b_{20} \neq 0,\left|a_{30}\right|+\left|b_{30}\right|>0$.

$$
\begin{aligned}
& \text { R: } a_{12} b_{12} a_{30} \neq 0, \\
& \text { I: } a_{30}=0, b_{30} \neq 0, \\
& \text { I: } a_{30} \neq 0, a_{12}=0, b_{12} \neq 0, \\
& \text { I: } a_{30} \neq 0, a_{12} \neq 0, b_{12}=0 .
\end{aligned}
$$

CASE 6. $\left|a_{11}\right|+\left|b_{11}\right|>0, b_{21} \neq 0,\left|a_{30}\right|+\left|b_{30}\right|>0$.

$$
\begin{aligned}
& \text { R: } a_{11} a_{30} b_{30} \neq 0, \\
& \text { I: } a_{11}=0, b_{11} \neq 0, \\
& \text { I: } a_{11} \neq 0, a_{30}=0, b_{30} \neq 0, \\
& \text { I: } a_{11} \neq 0, a_{30} \neq 0, b_{30}=0 .
\end{aligned}
$$

CASE 7. $\left|a_{11}\right|+\left|b_{11}\right|>0, b_{20} \neq 0,\left|a_{30}\right|+\left|b_{30}\right|>0$.

$$
\begin{aligned}
& \mathrm{R}: a_{30} a_{11} b_{11} \neq 0, \\
& \text { I: } a_{30}=0, b_{30} \neq 0, \\
& \text { I: } a_{30} \neq 0, a_{11}=0, b_{11} \neq 0, \\
& \text { I: } a_{30} \neq 0, a_{11} \neq 0, b_{11}=0 .
\end{aligned}
$$

The purpose of this section is to write sets of BC's equivalent to the BC's $\mathrm{I}(\mathrm{B})$ in Cases 1-7 respectively, and having as many 0's in their BM's as possible. After selecting the consistent further conditions, we note that if an element $a_{i}$ or $b_{i}$ of the BM is not zero, then the LC obtained by multiplying the $\mathrm{BC}$ corresponding to its row by the reciprocal of this nonzero element replaces it by the element 1 . Also, by adding multiples of this row to the preceding rows we do not change any coefficient of a higher order derivative, and so all elements above the 1 and in the same column can be replaced by the element 0 . The necessary LC's will not be stated, and the original notation will be kept for each element other than 0 or 1 . By proceeding in this way, we have proved

Theorem 1.2. Any three LI third order $B C$ 's $I(B)$ are given by linear $B F^{\prime} s$ expressible canonically by one of the following $B M^{\prime} s$, whose columns are ordered as in (0.2), or the BM's obtained from them by interchanging the letters $a$ and $b$ and also the " $a$ " and " $b$ " submatrices of each $B M$ : 
CAse 1 (a)

$\left[\begin{array}{llllll}0 & 0 & a_{10} & 1 & b_{11} & b_{10} \\ 1 & 0 & a_{20} & 0 & b_{21} & b_{20} \\ 0 & 1 & a_{30} & 0 & 0 & b_{30}\end{array}\right]$.

CAse 2 (a)

$\left[\begin{array}{llllll}0 & a_{11} & 0 & 1 & b_{11} & b_{10} \\ 1 & a_{21} & 0 & 0 & b_{21} & b_{20} \\ 0 & 0 & 1 & 0 & 0 & 0\end{array}\right]$.

CASE 3 (a)

$\left[\begin{array}{llllll}1 & 0 & a_{10} & 0 & 0 & b_{10} \\ 0 & 1 & a_{20} & 0 & 0 & b_{20} \\ 0 & 0 & a_{30} & 0 & 1 & b_{20}\end{array}\right]$.

CASE 4

$$
\left[\begin{array}{llllll}
a_{12} & a_{11} & a_{10} & b_{12} & b_{11} & b_{10} \\
0 & a_{21} & a_{20} & 0 & b_{21} & b_{20} \\
0 & 0 & a_{30} & 0 & 0 & b_{30}
\end{array}\right],
$$

(a)

$$
b_{12} b_{21} a_{30}+b_{12} a_{21} b_{30}+a_{12} b_{21} b_{30}=0 \text {. }
$$

CASE 5 (a)

$$
\begin{aligned}
& {\left[\begin{array}{llllll}
1 & a_{11} & 0 & 0 & b_{11} & 0 \\
0 & 0 & 0 & 0 & 0 & 1 \\
0 & 0 & 1 & 0 & 0 & 0
\end{array}\right] .} \\
& {\left[\begin{array}{llllll}
0 & 0 & 0 & 0 & 1 & b_{10} \\
0 & 1 & 0 & 0 & 0 & b_{20} \\
0 & 0 & 1 & 0 & 0 & 0
\end{array}\right] .}
\end{aligned}
$$

CAsE 6 (a)

$$
\left[\begin{array}{llllll}
0 & 1 & 0 & 0 & 0 & 0 \\
0 & 0 & 1 & 0 & 0 & 0 \\
0 & 0 & 0 & 0 & 0 & 1
\end{array}\right] \text {. }
$$

REMARK 1.2.1. In Case 4(a), the reason for selecting the indicated further condition will emerge from the discussion following Corollary 3.2.1. In all other cases, the (a) subcase has a shorter uninterrupted sequence of 0's proceeding up the successive columns of the " $a$ " submatrix of the BM than does the " $b$ " submatrix.

The identification of degenerate sets of BC's for $T_{3}-(D)$ depends upon its characteristic determinant $[C D, \Delta]$, and will be made at the end of the next section.

2. The CD and Green's function for $T_{n}(D)$ [9]. The CD for $T_{n}(D)$ is defined by

$$
\Delta\left(y_{1}, \cdots, y_{n}\right) \equiv \operatorname{det} U_{i}\left(y_{j}\right)
$$$$
(i, j=1, \cdots, n),
$$

where $y_{1}, \cdots, y_{n}$ are a fundamental set of solutions [FS] of $L(D) y=0$. The 
Green's function $G(x ; t)$ for $T_{n}(D)$ is given by

$$
G(x ; t)=Z(x ; t) / \Delta
$$

where $Z(x ; t)$ is the CD augmented by the $(n+1)$ th row $y_{1}(x), \cdots, y_{n}(x)$, $g(x ; t)$ and by the $(n+1)$ th column $U_{1}(g), \cdots, U_{n}(g), g(x ; t)$, and $g(x ; t)$ is a fundamental solution [fs] of $L(D) y=0$, that is, a solution (except at $x=t$ ) such that $\partial^{k-1} g / \partial x^{k-1}(k=1, \cdots, n-1)$ are continuous at $x=t$, but $\partial^{n-1} g / \partial x^{n-1}$ has a jump of $-1 / p_{0}(t)$ at $x=t$. The calculation of $\Delta$ and $Z$ will be simplified by the use of zero values for some of the quantities $y_{j}^{(k-1)}(0), y_{j}^{(k-1)}(1)$ $(j, k=1, \cdots, n)$, in accordance with the analysis which follows.

LemMA 2.1. If $y_{1}, \cdots, y_{n}$ are a FS for $L(D) y=0$, then $\Delta\left(y_{1}, \cdots, y_{n}\right)$ $=\sum_{k=1}^{{ }_{2 n} C_{n}} C_{k} \mu_{k}\left(y_{1}, \cdots, y_{n}\right)$, where $C_{k}$ is the $n \times n$ determinant whose columns are the $\kappa$ th combination of $n$ columns (in any one of the $n$ ! possible orders) of the $B M$ with the ith row $a_{i 0}, \cdots, a_{i, n-1}, b_{i 0}, \cdots, b_{i, n-1}$, and $\mu_{k}\left(y_{1}, \cdots, y_{n}\right)$ is the $n \times n$ determinant whose columns correspond to those of $C_{k}$ in the $W$ matrix with the ith row $y_{i}(0), \cdots, y_{i}^{(n-1)}(0), y_{i}(1), \cdots, y_{i}^{(n-1)}(1)$ (and are in the order corresponding to that of $C_{x}$ ).

Proof. This lemma is a special case (involving two $n \times 2 n$ matrices) of a theorem proved in the treatise by Muir [10].

COROLLARY 2.1. If the linear transformation [LT] of the FS $y_{\gamma k}(x)$ into the FS $y_{j}(x)$ is given by $y_{j}(x)=\sum_{k=1}^{n} c_{j k} y_{\gamma k}(x)(j=1, \cdots, n)$, then $\mu_{k}\left(y_{1}, \cdots, y_{n}\right)$ $=\left(\operatorname{det} c_{j k}\right) \mu_{k}\left(y_{\gamma 1}, \cdots, y_{\gamma n}\right)$.

Proof. This is an instance of the usual rule for multiplying two determinants.

LemMA 2.2. $Z(x ; t)=\sum_{k=1}^{{ }_{2 n} C_{n}} C_{k} M_{k}(x ; t)$, where $C_{k}$ has the same meaning as in Lemma 2.1, and $M_{\kappa}(x ; t)$ is the $(n+1) \times(n+1)$ determinant formed by first augmenting the $W$ matrix by the $(n+1)$ th row $g(0), \cdots, g^{(n-1)}(0), g(1), \cdots$, $g^{(n-1)}(1), g(x ; t)$ and the $(2 n+1)$ th column $y_{1}(x), \cdots, y_{n}(x), g(x ; t)$, and then taking as the first $n$ columns those corresponding to the respective columns of $\mu_{\mathrm{k}}$, and $y_{1}(x), \cdots, y_{n}(x), g(x ; t)$ as the $(n+1)$ th column.

Proof. Expanding the defining determinant for $Z(x ; t)$ by minors of its last row gives

$$
Z(x ; t)=\left[\sum_{j=1}^{n}(-1)^{n+1+j} y_{j}(x) \Delta_{j}\right]+(-1)^{2 n+2} g(x ; t) \Delta,
$$

where $\Delta_{j}$ is $\Delta$ with its $j$ th column deleted and $U_{1}(g), \cdots, U_{n}(g)$ as its $n$th column. The theorem used in proving Lemma 2.1 is also applicable to $\Delta_{j}$ and gives, after interchanging summations over $j$ and $\kappa$, 


$$
Z(x ; t)=\sum_{k=1}^{2 n} C_{k}\left[\left(\sum_{j=1}^{n}(--1)^{n+1+i} y_{j}(x) \mu_{k j}\right)+(-1)^{2 n+2} g(x ; t) \mu_{k}\right],
$$

where $\mu_{k j}$ transposed is $\mu_{k}$ with the $j$ th row written as the $n$th and $g$ replacing $y_{j}$ in it. The proof is completed by noting that the expression multiplying $C_{k}$ in (2.2.2) is precisely the expansion of $M_{k}(x ; t)$ by minors of its last column, since $\mu_{k j}$ is the transpose of the minor of $y_{j}(x)$ in this expansion.

COROLlaRY 2.2.1. If the LT of the FS $y_{\gamma k}(x)$ into the FS $y_{j}(x)$ is given by $y_{j}(x)=\sum_{k=1}^{n} c_{j k} y_{\gamma k}(x)(j=1, \cdots, n)$, then $M_{k}(x ; t)=\left(\operatorname{det} c_{j k}\right) M_{\gamma k}(x ; t)$ where $M_{\gamma \kappa}$ is obtained from $M_{\alpha}$ by replacing the FS $y_{j}$ by the FS $y_{\gamma j}$.

Proof. By the rule for multiplying determinants, each minor of the last row (the $g$-row) of $M_{k}$ equals det $c_{j k}$ times the corresponding minor of $M_{\gamma k \text {. }}$

COROLlaRY 2.2.2. $M_{k}(x ; t)$ is invariant under the change from one $f s g(x ; t)$ to another.

Proof. Since any two fs's differ only by a LC of the $y_{j}(x)$, such a change merely adds to the last row of $M_{\mathrm{k}}$ a LC of the other rows.

REMARK 2.2.2. The convenient fs's will be $g_{a}(x ; t), g_{b}(x ; t)$ defined by $g_{a}=0$ for $x<t, g_{a}=y(x ; t)$ for $x>t ; g_{b}=-y(x ; t)$ for $x<t, g_{b}=0$ for $x>t$; $y(x ; t)$ is the solution of $L(D) y=0$ such that $\partial^{k-1} y / \partial x^{k-1}=0(k=1, \cdots, n-1)$ at $x=t$, and $\partial^{n-1} y / \partial x^{n-1}=-1 / p_{0}(t)$ at $x=t$.

In order to use two convenient FS's, we need the following

Definition. The FS $y_{j}(x)$ is a normalized FS [NFS] at $x=x_{0}$ if $y_{j}^{(k-1)}\left(x_{0}\right)$ $=\delta_{j k}$, where $\delta_{j k}$ is the Kronecker delta.

REMARK 2.3.0. If $y_{j}(x)$ is any FS, then the NFS at $x=x_{0}$ is $W_{j}\left(x ; x_{0}\right) / W\left(x_{0}\right)$, where $W_{j}\left(x ; x_{0}\right)$ is the Wronskian $W\left(x_{0}\right)$ with its $j$ th row replaced by $y_{1}(x), \cdots, y_{n}(x)$.

LEMMA 2.3. If $y_{a j}(x), y_{b j}(x)$ are NFS's at $x=0$ and $x=1$ respectively, then $y_{b j}(x)=\sum_{k=1}^{n} y_{b j}^{(k-1)}(0) y_{a k}(x)$ and $y_{a j}(x)=\sum_{k=1}^{n} y_{a j}^{(k-1)}(1) y_{b k}(x)$.

Proof. These relations result from the LT's $y_{b j}(x)=\sum_{k-1}^{k} c_{j k} y_{a k}(x)$ and $y_{a j}(x)=\sum_{k=1}^{n} C_{j k} y_{b k}(x)$ by differentiating $k-1$ times and then substituting $x=0$ and $x=1$ respectively.

REMARK 2.3.1. The matrices of these mutually inverse LT's are the transposed Wronskians $\left[W_{b}(0)\right]^{\prime}$ and $\left[W_{a}(1)\right]^{\prime}$ respectively, and therefore $W_{b}(0) W_{a}(1)=1$. If $p_{1}(x) \equiv 0$, by Abel's identity $W_{b}(0)=W_{a}(1)=1$.

REMARK 2.3.2. If the coefficients of $L(D) y$ are constant, then $y_{a j}(x-1)$ $=y_{b j}(x), y_{b j}(x+1)=y_{a j}(x)$, and $y(x ; t)=\left[-1 / p_{0}(t)\right] y_{a n}(x-t)$.

REMARK 2.3.3. The results of this section apply to the interval $[a, b]$ if the symbols " 0 " and " 1 " appearing explicitly as function arguments are replaced by $a$ and $b$ respectively, and the symbol " 1 " in $x-1$ and $x+1$ in Remark 2.3.2 is replaced by $b-a$, and $x-t$ is replaced by $x-(t-a)$ in the same remark. 
By the foregoing analysis, beginning with I.emma 2.1, we have proved

TheOREM 2.4. Let $L(D) y$ be given by $(0.1)$, let $y_{a j}(x), y_{b j}(x)$, $y_{t j}(x)(j=1, \cdots, n)$ be NFS's of $L(D) y=0$ at $x=a, x=b, x=t$ respectively, and let $G(x ; t)=Z(x ; t) / \Delta$ be the Green's function for $L(D) y=0$ and the associated $L I B C$ 's (0.2), with the arguments 0 and 1 replaced by $a$ and $b$ respectively. Let $y(x ; t)=-y_{\text {tn }}(x) / p_{0}(t)$, and let $\left[W_{a}(b)\right]^{\prime},\left[W_{b}(a)\right]^{\prime}$ denote the respective transposed Wronskian matrices of the $y_{a j}$ for $x=b$ and the $y_{b j}$ for $x=a$. Then from the matrices

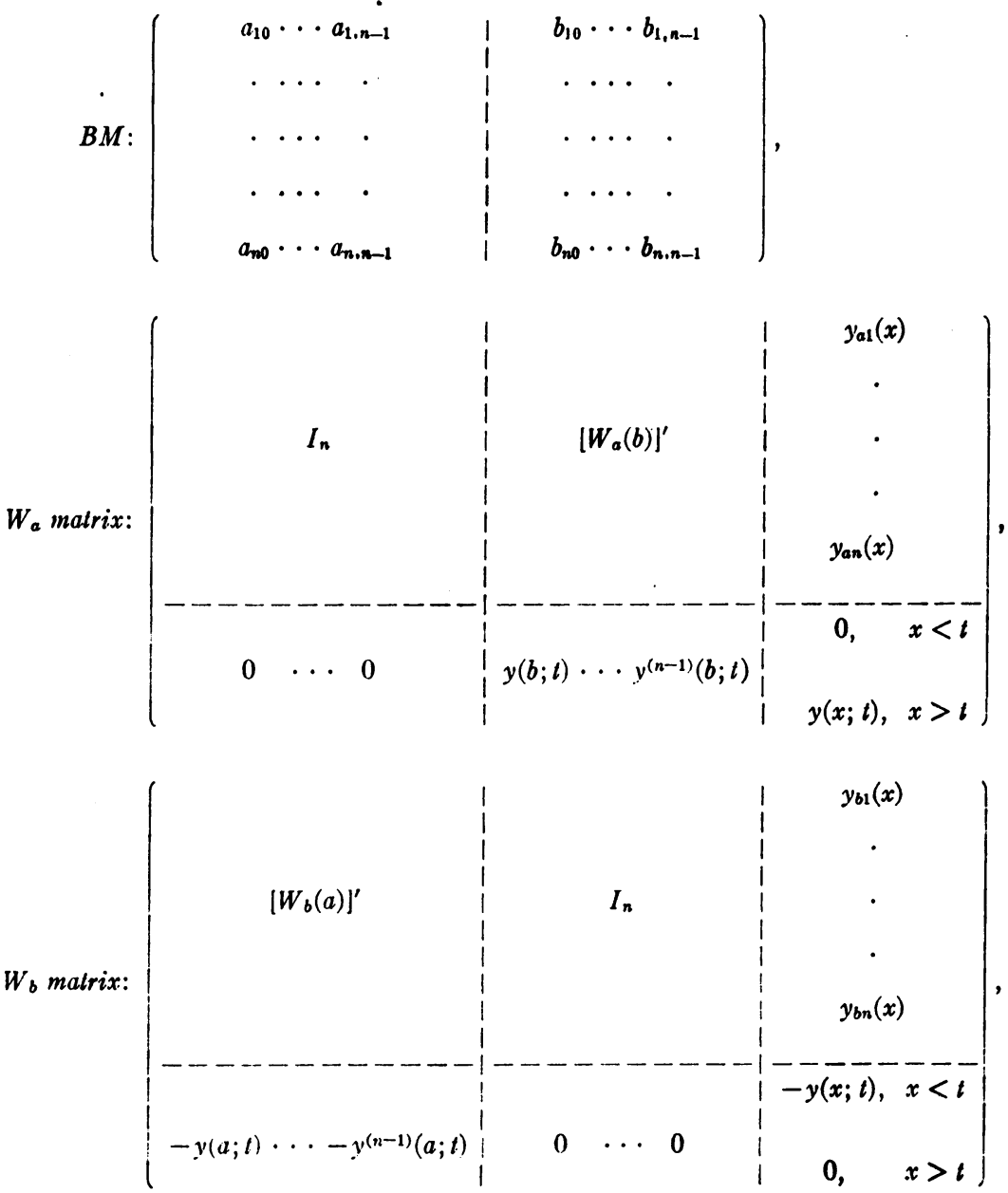

where the derivatives indicated in the augmenting rows are with respect to $x, \Delta_{a}$ is obtained by taking each determinant $C_{\kappa}\left(\kappa=1, \cdots,{ }_{2 n} C_{n}\right)$, whose columns are one of the ${ }_{2 n} C_{n}$ distinct sets of $n$ columns of the $B M$, and multiplying it either by the corresponding $n \times n$ determinant $\mu_{a x}$ formed from the $W_{a}$ matrix or by $W_{a}(b)$ times the corresponding $n \times n$ determinant $\mu_{b x}$ formed from the $W_{b}$ matrix, and 
then summing over all $\kappa ; Z_{a}(x ; t)$ is obtained in the same way, but with $\mu_{a x}$ and $\mu_{b \mathrm{k}}$ replaced by the corresponding augmented determinants $M_{a x}$ and $M_{b \mathrm{k}}$ respectively.

REMARK 2.4.1. The conclusion of Theorem 2.4 remains true when the symbols $a$ and $b$ are interchanged in it, beginning with $\Delta_{a}$.

REMARK 2.4.2. The $W_{b}$ matrix is used when $C_{k}$ has more $b_{i, j-1}$ columns than $a_{i, j-1}$ columns.

As a preliminary to the calculation of $\Delta$ and $Z$ for the eigenvalue equation [EE] $L(D) y-\lambda y=0$ for $T_{3}^{-}(D)$, the following formulae involving trigonometric functions of order $n\left[u_{j}\right][6]$, which satisfy $y^{(n)}+y=0$, are presented. Here and subsequently, $\omega_{l}=e^{i(2 l-1) \pi / n}(l=1, \cdots, n)$.

General formulae:

$$
\begin{aligned}
u_{j}(z) & =\frac{1}{n} \sum_{l=1}^{n} \omega_{l}^{1-j} e^{z \omega_{l}} & & (j=1, \cdots, n), \\
u_{j+n}(z) & =-u_{j}(z) & & (j \text { any integer }), \\
u_{j}^{(s)}(z) & =u_{j-\imath}(z) & & (j, s \text { any integers), } \\
u_{j}^{(o-1)}(0) & =\delta_{j s} & & (j, s=1, \cdots, n), \\
u_{j}(z+w) & =\sum_{s=1}^{n} u_{j-o+1}(w) u_{s}(z) & & (j \text { any integer }), \\
u_{j}(z) & =\sum_{r=0}^{\infty} \frac{(-1)^{r} z^{n r+j-1}}{(n r+j-1) !} & & (j=1, \cdots, n), \\
u_{j}\left(\omega_{1}^{2} z\right) & =\omega_{1}^{2(j-1)} u_{j}(z) & & (j \text { any integer }) .
\end{aligned}
$$

Special formulae for $n=3$ :

$$
\begin{aligned}
u_{j}(z)=\frac{1}{3}(-1)^{j-1} e^{-z} & +\frac{2}{3} e^{z / 2} \cos \left[-(j-1) \frac{\pi}{3}+\frac{3^{1 / 2}}{2} z\right] \\
u_{1}(-z) & =u_{1}^{2}(z)+u_{2}(z) u_{3}(z) \\
u_{2}(-z) & =-u_{3}^{2}(z)-u_{1}(z) u_{2}(z) \\
u_{3}(-z) & =u_{2}^{2}(z)-u_{3}(z) u_{1}(z)
\end{aligned}
$$

Let $\lambda=\rho^{3}$. Formula (2.5.04), Remarks 2.3.2 and 2.3.1 give, respectively, $y_{a j}(x)=\rho^{1-i} u_{j}(\rho x), \quad y_{b j}(x)=\rho^{1-i} u_{j}(\rho(x-1)), \quad W_{a}(1)=1$. Also, $y(x ; t)$ $=\rho^{-2} u_{3}(\rho(x-t))$. The BM and determinants formed from it will be designated by writing only one row, with the subscript $i$ omitted. Using (2.5.03) and (2.5.04) gives the desired matrices: 


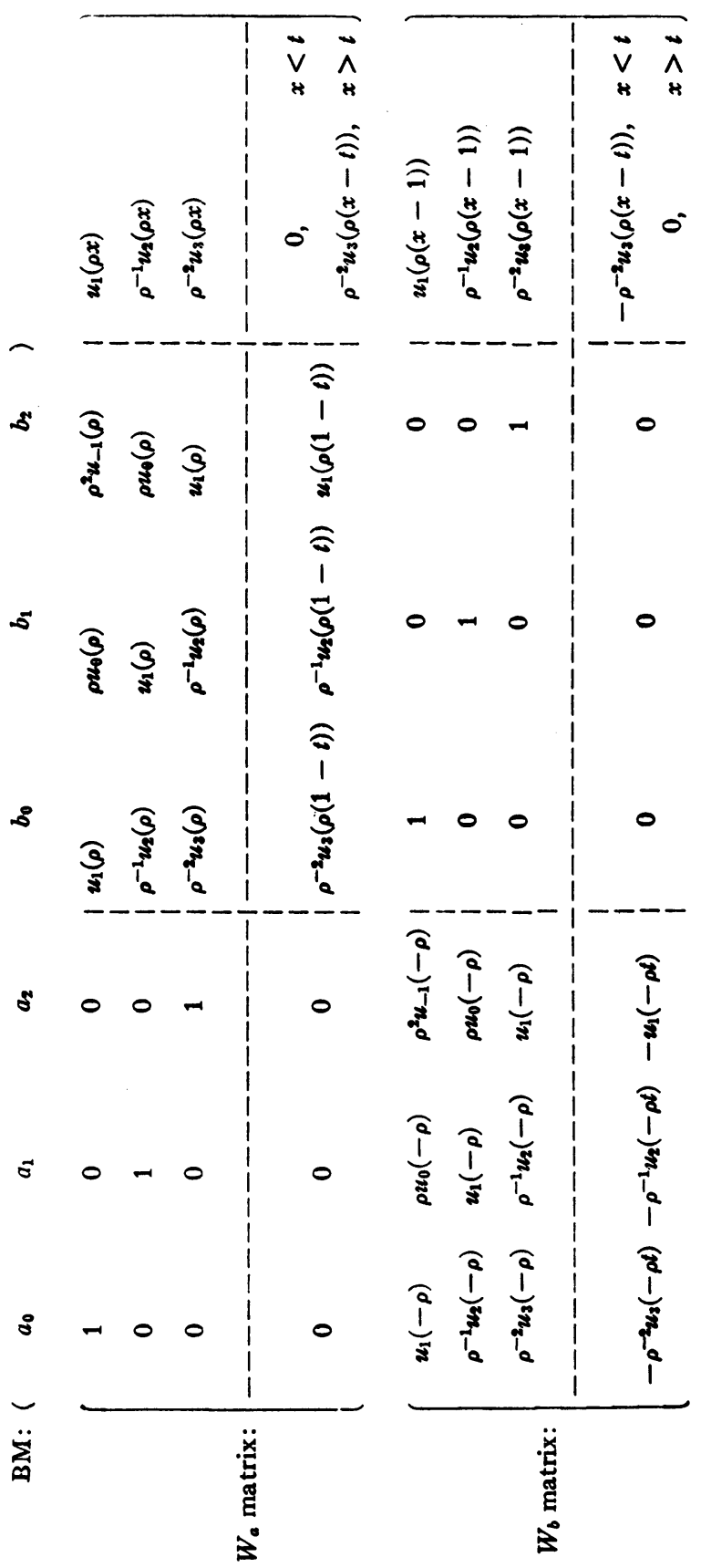


By Theorem 2.4, Remark 2.4.2, and Formula (2.5.02), it follows easily that

$$
\begin{aligned}
\rho^{3} \Delta(\rho)= & -\left|a_{2} a_{1} a_{0}\right| \rho^{3}+\left(-\left|a_{2} a_{1} b_{0}\right| \rho^{3}+\left|a_{2} a_{0} b_{1}\right| \rho^{3}-\left|a_{1} a_{0} b_{2}\right| \rho^{3}\right) u_{1}(\rho) \\
& +\left(\left|a_{2} a_{1} b_{2}\right| \rho^{5}+\left|a_{2} a_{0} b_{0}\right| \rho^{2}-\left|a_{1} a_{0} b_{1}\right| \rho^{2}\right) u_{2}(\rho) \\
& +\left(\left|a_{2} a_{1} b_{1}\right| \rho^{4}-\left|a_{2} a_{0} b_{2}\right| \rho^{4}-\left|a_{1} a_{0} b_{0}\right| \rho\right) u_{3}(\rho) \\
& +\left(-\left|a_{0} b_{2} b_{1}\right| \rho^{3}+\left|a_{1} b_{2} b_{0}\right| \rho^{3}-\left|a_{2} b_{1} b_{0}\right| \rho^{3}\right) u_{1}(-\rho) \\
& +\left(\left|a_{2} b_{2} b_{1}\right| \rho^{5}+\left|a_{0} b_{2} b_{0}\right| \rho^{2}-\left|a_{1} b_{1} b_{0}\right| \rho^{2}\right) u_{2}(-\rho) \\
& +\left(\left|a_{1} b_{2} b_{1}\right| \rho^{4}-\left|a_{2} b_{2} b_{0}\right| \rho^{4}-\left|a_{0} b_{1} b_{0}\right| \rho\right) u_{3}(-\rho)-\left|b_{2} b_{1} b_{0}\right| \rho^{3},
\end{aligned}
$$

\begin{tabular}{|c|c|c|}
\hline \multirow{2}{*}{$\begin{array}{l}\text { Coefficient } \\
C_{\kappa \rho^{m_{k}}}\end{array}$} & \multicolumn{2}{|c|}{ Multiplier $M_{k \rho}-m_{k}$} \\
\hline & $(x<t)$ & $(x>t)$ \\
\hline$\left|a_{2} a_{1} a_{0}\right| \rho^{-2}$ & 0 & $-u_{3}(\rho(x-t))$ \\
\hline$\left|a_{2} a_{1} b_{0}\right| \rho^{-2}$ & $u_{3}(\rho(1-t)) u_{1}(\rho x)$ & $u_{3}(\rho(1-t)) u_{1}(\rho x)-u_{3}(\rho(x-t)) u_{1}(\rho)$ \\
\hline$\left|a_{2} a_{0} b_{1}\right| \rho^{-2}$ & $-u_{2}(\rho(1-t)) u_{2}(\rho x)$ & $-u_{2}(\rho(1-t)) u_{2}(\rho x)+u_{3}(\rho(x-t)) u_{1}(\rho)$ \\
\hline$\left|a_{1} a_{0} b_{2}\right| \rho^{-2}$ & $u_{1}(\rho(1-t)) u_{3}(\rho x)$ & $u_{1}(\rho(1-t)) u_{3}(\rho x)-u_{3}(\rho(x-t)) u_{1}(\rho)$ \\
\hline$\left|a_{2} a_{1} b_{2}\right|$ & $u_{1}(\rho(1-t)) u_{1}(\rho x)$ & $u_{1}(\rho(1-t)) u_{1}(\rho x)+u_{2}(\rho(x-t)) u_{2}(\rho)$ \\
\hline$\left|a_{2} a_{0} b_{0}\right| \rho^{-3}$ & $-u_{3}(\rho(1-t)) u_{2}(\rho x)$ & $-u_{3}(\rho(1-t)) u_{2}(\rho x)+u_{3}(\rho(x-t)) u_{2}(\rho)$ \\
\hline$\left|a_{1} a_{0} b_{1}\right| \rho^{-3}$ & $u_{2}(\rho(1-t)) u_{3}(\rho x)$ & $u_{2}(\rho(1-t)) u_{3}(\rho x)-u_{3}(\rho(x-t)) u_{2}(\rho)$ \\
\hline$\left|a_{2} a_{1} b_{1}\right| \rho^{-1}$ & $u_{2}(\rho(1-t)) u_{1}(\rho x)$ & $u_{2}(\rho(1-t)) u_{1}(\rho x)+u_{3}(\rho(x-t)) u_{3}(\rho)$ \\
\hline$\left|a_{2} a_{0} b_{2}\right| \rho^{-1}$ & $-u_{1}(\rho(1-t)) u_{2}(\rho x)$ & $-u_{1}(\rho(1-t)) u_{2}(\rho x)-u_{3}(\rho(x-t)) u_{3}(\rho)$ \\
\hline$\left|a_{1} a_{0} b_{0}\right|_{\rho^{-4}}$ & $u_{3}(\rho(1-t)) u_{3}(\rho x)$ & $u_{3}(\rho(1-t)) u_{3}(\rho x)-u_{3}(\rho(x-t)) u_{3}(\rho)$ \\
\hline$\left|a_{0} b_{2} b_{1}\right| \rho^{-2}$ & $-u_{3}(-\rho t) u_{1}(\rho(x-1))+u_{3}(\rho(x-t)) u_{1}(-\rho)$ & $-u_{3}(-\rho t) u_{1}(\rho(x-1))$ \\
\hline$\left|a_{1} b_{2} b_{0}\right| \rho^{-2}$ & $u_{2}(-\rho t) u_{2}(\rho(x-1))-u_{3}(\rho(x-t)) u_{1}(-\rho)$ & $u_{2}(-\rho t) u_{2}(\rho(x-1))$ \\
\hline$\left|a_{2} b_{1} b_{0}\right| \rho^{-2}$ & $-u_{1}(-\rho t) u_{3}(\rho(x-1))+u_{3}(\rho(x-t)) u_{1}(-\rho)$ & $-u_{1}(-\rho t) u_{3}(\rho(x-1))$ \\
\hline$\left|a_{2} b_{2} b_{1}\right|$ & $-u_{1}(-\rho t) u_{1}(\rho(x-1))-u_{3}(\rho(x-t)) u_{2}(-\rho)$ & $-u_{1}(-\rho t) u_{1}(\rho(x-1))$ \\
\hline$\left|a_{0} b_{2} b_{0}\right|_{\rho^{-3}}$ & $u_{3}(-\rho t) u_{2}(\rho(x-1))-u_{3}(\rho(x-t)) u_{2}(-\rho)$ & $u_{3}(-\rho t) u_{2}(\rho(x-1))$ \\
\hline$\left|a_{1} b_{1} b_{0}\right| \rho^{-3}$ & $-u_{2}(-\rho t) u_{3}(\rho(x-1))+u_{3}(\rho(x-t)) u_{2}(-\rho)$ & $-u_{2}(-\rho t) u_{3}(\rho(x-1))$ \\
\hline$\left|a_{1} b_{2} b_{1}\right| \rho^{-1}$ & $-u_{2}(-\rho t) u_{1}(\rho(x-1))-u_{3}(\rho(x-t)) u_{3}(-\rho)$ & $-u_{2}(-\rho t) u_{1}(\rho(x-1))$ \\
\hline$\left|a_{2} b_{2} b_{0}\right| \rho^{-1}$ & $u_{1}(-\rho t) u_{2}(\rho(x-1))+u_{3}(\rho(x-t)) u_{3}(-\rho)$ & $u_{1}(-\rho t) u_{2}(\rho(x-1))$ \\
\hline$\left|a_{0} b_{1} b_{0}\right| \rho^{-4}$ & $-u_{3}(-\rho t) u_{3}(\rho(x-1))+u_{3}(\rho(x-t)) u_{3}(-\rho)$ & $-u_{3}(-\rho t) u_{3}(\rho(x-1))$ \\
\hline$\left|b_{2} b_{1} b_{0}\right| \rho^{-2}$ & $u_{3}(\rho(x-t))$ & 0 \\
\hline
\end{tabular}

where the columns for each $C_{k}$ have been ordered as in the BF's (0.2), and that the terms $C_{k} M_{k}$ of $Z(x ; t, \rho)$ are as indicated in Table 2.5.1, which follows. Each power $\rho^{m_{k}}$ occurring as a factor of $M_{k}$ has been associated with $C_{k}$ for convenience in the work of $\$ 4$.

TABLE 2.5.1. TERMS OF $Z(x ; t, \rho)$ FOR $T_{\overline{3}}^{-}(D)$ 
REMARK 2.5.1. Using only the NFS $\rho^{1-j} u_{j}(\rho x)$ entails more calculation (although less than the FS $e^{\rho \omega_{i} x}$ ), and requires (2.5.05) and (2.5.09) for simplification.

In order to identify and exclude degenerate cases, (2.5.01) is substituted in (2.5.1). Since $\omega_{l}^{3}=-1$, there follows

$$
3 \rho^{3} \Delta(\rho)=C \rho^{3}+\sum_{l=1}^{3} P_{a}\left(\rho \omega_{l}\right) e^{\rho \omega_{l}}+\sum_{l=1}^{8} P_{b}\left(\rho \omega_{l}\right) e^{-\rho \omega_{l}},
$$

where

$$
\begin{aligned}
C \equiv & -3\left(\left|a_{2} a_{1} a_{0}\right|+\left|b_{2} b_{1} b_{0}\right|\right) \\
P_{a}\left(\rho \omega_{l}\right) \equiv & \left|a_{2} a_{1} b_{2}\right|\left(\rho \omega_{l}\right)^{5}+\left(\left|a_{2} a_{1} b_{1}\right|-\left|a_{2} a_{0} b_{2}\right|\right)\left(\rho \omega_{l}\right)^{4} \\
& +\left(\left|a_{2} a_{1} b_{0}\right|-\left|a_{2} a_{0} b_{1}\right|+\left|a_{1} a_{0} b_{2}\right|\right)\left(\rho \omega_{l}\right)^{3} \\
& +\left(-\left|a_{2} a_{0} b_{0}\right|+\left|a_{1} a_{0} b_{1}\right|\right)\left(\rho \omega_{l}\right)^{2}+\left|a_{1} a_{0} b_{0}\right|\left(\rho \omega_{l}\right), \\
P_{b}\left(\rho \omega_{l}\right) \equiv & \left|a_{2} b_{2} b_{1}\right|\left(\rho \omega_{l}\right)^{5}+\left(\left|a_{1} b_{2} b_{1}\right|-\left|a_{2} b_{2} b_{0}\right|\right)\left(\rho \omega_{l}\right)^{4} \\
& +\left(\left|a_{0} b_{2} b_{1}\right|-\left|a_{1} b_{2} b_{0}\right|+\left|a_{2} b_{1} b_{0}\right|\right)\left(\rho \omega_{l}\right)^{3} \\
& +\left(-\left|a_{0} b_{2} b_{0}\right|+\left|a_{1} b_{1} b_{0}\right|\right)\left(\rho \omega_{l}\right)^{2}+\left|a_{0} b_{l} b_{0}\right|\left(\rho \omega_{l}\right) .
\end{aligned}
$$

The BC's (0.2) are called degenerate if the associated $\mathrm{EE} L(D) y=\lambda y$ has only a finite number of solutions satisfying them. A necessary condition for the exclusion of degenerate BC's is the existence of infinitely many zeros $\rho_{n}$ of (2.6.1), making infinitely many $\rho_{n}^{3}$ simple zeros of $\Delta$ (Remark 3.0.6) and poles of $G$ [9]. The sufficiency of this condition follows from the existence of the corresponding eigensolutions $u_{j}\left(\rho_{n} x\right)$. By complex variable theory, $\Delta(\rho)$ for $L(D)=-D^{3}$ and $(0.2)$ (which include $T_{3}^{-}(D)$ as a special case) has infinitely many zeros if and only if the coefficients of $P_{a}\left(\rho \omega_{l}\right)$ or $P_{b}\left(\rho \omega_{l}\right)$ are not all zero. Therefore, we have established

Theorem 2.6. The $B C^{\prime}$ 's (0.2) associated with $L(D)=-D^{3}$ are degenerate if and only if all coefficients of $P_{a}\left(\rho \omega_{l}\right)$ and $P_{b}\left(\rho \omega_{l}\right)$, as given by (2.6.1), vanish.

In the next section, some general results will be obtained from which information about the spectrum of $T_{3}^{-}(D)$ will then follow by specialization.

3. Exponential sums and the spectrum of $T_{3}^{-}(D)$. The necessary material by Schwengeler [13] is summarized as follows:

Remark 3.0.1. An exponential sum $[\mathrm{ES}, F(z)]$ is defined by

$$
F(z) \equiv \sum_{\nu=1}^{N} P_{\nu}(z) e^{a, z}
$$

in which the $a_{\nu}$ are distinct complex constants and the $P_{\nu}(z)$ are polynomials in the complex variable $z$ which are not identically zero. $F(z)$ is an entire 
transcendental function whose zeros have no finite limit point in the complex plane.

Remark 3.0.2. The indicator diagram [ID] is the unique smallest convex polygon such that none of the exponent points [EP's] $a_{v}^{*}$ lie outside of it. Each vertex is an EP.

REMARK 3.0.3. As arg $z$ varies from 0 to $2 \pi$, each vertex of the ID assumes the role of representative of the prevalent term [PT] of $F(z)$; that is, the term of largest absolute value as $|z| \rightarrow \infty$. A transition direction $\phi_{v}$ is the direction of the outgoing perpendicular to a side $S$ of the ID. In the direction $\phi_{w}$, each EP on $S$ corresponds to a PT. This means that the terms of $F(z)$ of largest absolute value in the direction $\phi_{w}$ correspond to those exponentials $e^{a_{\nu} z}$ whose EP's lie on $S$.

REMARK 3.0.4. In an angular region including the boundary sides, which are rays from $O$ in the respective directions of the outgoing normals to two consecutive sides of the indicator diagram, that term of $F(z)$ is prevalent which has the intersection of these two sides as its EP. An EP in the interior of the ID cannot correspond to a PT. More specifically, if the projection of the EP $a_{j}^{*}$ on a ray in the direction $\phi$ is algebraically further from $O$ than the projection of the EP $a_{k}^{*}$, then $\exists$ a positive constant $c \in P_{k}(z) e^{a_{k z}} / P_{j}(z) e^{a_{j z}}$ $=O\left(e^{-c|z|}\right)$ as $|z| \rightarrow \infty$ and $\arg z=\phi$.

REMARK 3.0.5. The zeros of $F(z)$, with finitely many possible exceptions [w.f.m.p.e.], lie in disjoint angular regions [AR's] (each with vertex at $O$ and of arbitrarily small angle) given by $\phi_{w}-\epsilon<\phi<\phi_{w}+\epsilon$, with $\epsilon>0$.

REMARK 3.0.6. When the only EP's on $S$ are the two vertices, the number $N_{w}(r)$ of zeros satisfying $0<|z| \leqq r$ in the AR corresponding to $\phi_{w}$ is given by $N_{w}(r)=r|S| / 2 \pi+O(1)$, where $|S|$ is the length of $S$. These zeros, w.f.m.p.e., are simple, and are given by

$$
z_{n}=\left[2 n \pi+i\left(m_{1}-m_{2}\right) \log (2 n \pi /|S|)+O(1)\right] e^{i \phi_{w}} /|S|,
$$

where $m_{1}, m_{2}$ are the respective degrees of the polynomials $P_{1}(z), P_{2}(z)$, and $a_{1}^{*}, a_{2}^{*}$ are the vertices on $S$ ordered so that $\phi_{w}=\pi / 2+\arg \left(a_{1}^{*}-a_{2}^{*}\right)$. The principal value of $\log (2 n \pi /|S|)$ is used.

To extend (3.0.6) through explicit terms which are $O\left(n^{-p} \log ^{p} n\right)$, with $p$ an arbitrary positive integer, (3.0.1) is transformed by means of the identity

$$
\alpha+\beta \equiv 2(\alpha \beta)^{1 / 2} \cos \left(\frac{i}{2} \log \frac{\alpha}{\beta}\right),
$$

with $\alpha=P_{1}(z) e^{a_{1} z}, \beta=P_{2}(z) e^{a_{2}}$. By Remark 3.0.4, with $j=1,2$, and $k=3, \cdots, N$, dividing $F(z)=0$ by $2(\alpha \beta)^{1 / 2}$ gives $\cos [(i / 2) \log (\alpha / \beta)]=\psi$, where $\psi \rightarrow 0$ exponentially as $|z| \rightarrow \infty$, for $z$ in the AR of Remark 3.0.5. We substitute $(i / 2) \log (\alpha / \beta)= \pm(n-1 / 2) \pi-\theta$, where $|\operatorname{Re} \theta|<\pi / 2$ and the positive integer $n$ is large enough, to ensure this (i.e., makes $|\psi|<1$ ). Thus, 
$\pm(-1)^{n-1} \sin \theta=\psi$. Therefore, $\theta \rightarrow 0$ and $\theta=O(\sin \theta)=O(\psi)$. From $(i / 2) \log (\alpha / \beta)=(i / 2)\left[\log \left(P_{1}(z) / P_{2}(z)\right)+\left(a_{1}-a_{2}\right) z\right], \phi_{w}=\pi / 2+\arg \left(a_{1}^{*}-a_{2}^{*}\right)=$ $\pi / 2-\arg \left(a_{1}-a_{2}\right)$ and $\arg z=\phi_{\omega}+\chi$, where $|\chi|<\epsilon$ by Remark 3.0.5, it follows that $\arg \left[(i / 2)\left(a_{1}-a_{2}\right) z\right]=\pi+\chi$ and that only the lower sign is applicable in the expressions $\pm(n-1 / 2) \pi$ and $\pm(-1)^{n-1} \sin$. Therefore, as $n \rightarrow \infty$

$$
\frac{i}{2}\left[\log \left(P_{1}(z) / P_{2}(z)\right)+\left(a_{1}-a_{2}\right) z\right]=-\left(n-\frac{1}{2}\right) \pi-\theta .
$$

Let $P_{\nu}(z)=\sum_{s=0}^{m_{\nu}} C_{\nu s} z^{m_{\nu}-s}$, and $C_{\nu 0} \neq 0(\nu=1, \cdots, N)$. Substituting $z=w e^{i \phi_{w}} /\left|a_{1}-a_{2}\right|$ in (3.1.2) transforms it into

$$
w=(2 n-1) \pi+i \log \left(Q_{1}(w) / Q_{2}(w)\right)+2 \theta,
$$

where $Q_{\nu}=\sum_{s=0}^{m_{\nu}} K_{\nu s} w^{m_{\nu}-s}(\nu=1,2)$, and $K_{\nu s}=\left(e^{i \phi_{w}} /\left|a_{1}-a_{2}\right|\right)^{m_{\nu}-s} C_{\nu s}$. For the iteration procedure which is to follow, the leading terms of $Q_{1}$ and $Q_{2}$ are factored out, thus giving $Q_{\nu}=K_{\nu 0} w^{m_{\nu}}\left(1+\epsilon_{\nu}\right)$, where $\epsilon_{\nu}=\sum_{s=1}^{m_{\nu}} c_{\nu s} w^{-s}$ with $c_{\nu s}=K_{\nu s} / K_{\nu 0}$ when $m_{\nu}>0$ but is assumed replaced by 0 when $m_{\nu}=0$. Thus,

$$
w=2 n \pi+i m \log w+c+\delta+2 \theta,
$$

where $m=m_{1}-m_{2}, c=-\pi+i \log \left(K_{10} / K_{20}\right)$, and $\delta=i \log \left[\left(1+\epsilon_{1}\right) /\left(1+\epsilon_{2}\right)\right]$. Taking logs and reciprocals respectively, after factoring out $2 n \pi$ in $a s y m p-$ totic expansions [AE's] of the form $w=2 n \pi+\eta$, which occur in the following analysis, lead to

$$
\begin{aligned}
\log w & =\log n+\log 2 \pi+\log (1+\xi), \\
w^{-s} & =(1+\xi)^{-8}(2 n \pi)^{-s}
\end{aligned}
$$

respectively, where $\xi=\eta / 2 n \pi$. Iteration in (3.1.4), with the aid of the respective Taylor polynomials [TP's] for $(1+\xi)^{-8}, \log (1+\xi), \log \left(1+\epsilon_{\nu}\right)(\nu=1,2)$, and then induction, will result in an asymptotic expansion up to an $O\left(n^{-p-1} \log ^{p+1} n\right)$ error term, with $p$ an arbitrary positive integer, as $n \rightarrow \infty$. There are three cases: Case (1) $m_{1} \neq m_{2}$; Case (2) $m_{1}=m_{2}, P_{1} \not \equiv P_{2}$; Case (3) $P_{1} \equiv P_{2}$.

CASE (1). $m_{1} \neq m_{2}$. The zeroth AE's for $\delta$ and $w$, indicated by using the superscript ( 0$)$, are defined to be those whose explicit terms of smallest order are $O(1)$ but not $o(1)$. Thus, $\delta^{(0)}=O\left(n^{-1}\right)$. From $w=2 n \pi+O(\log n)$, we get $w^{(0)}=2 n \pi+i m \log 2 n \pi+c+O\left(n^{-1} \log n\right)$, since $O[\log (1+\xi)]=O(\xi)$ $=O\left(n^{-1} \log n\right)$. By the definitions of $c$ and $K_{v s}$, Schwengeler's term indicated by " $O(1)$ " in (3.0.6) equals $-m \phi_{w}-\pi+i \log \left(C_{10} / C_{20}\right)+O\left(n^{-1} \log n\right)$. The first $\mathrm{AE} \delta^{(1)}$ is obtained from $w^{(0)}$, as follows: $\delta^{(1)}=i\left(c_{11}-c_{21}\right)\left(w^{(0)}\right)^{-1}+O\left(n^{-2}\right)$ $=i\left(c_{11}-c_{21}\right)[1+O(\xi)](2 n \pi)^{-1}+O\left(n^{-2}\right)=i\left(c_{11}-c_{21}\right)(2 n \pi)^{-1}+O\left(n^{-2} \log n\right)$. The first $\mathrm{AE} w^{(1)}$ is obtained from $w^{(0)}$ and $\delta^{(1)}$. as follows: 


$$
\begin{aligned}
w^{(1)}= & 2 n \pi+i m \log w^{(0)}+c+\delta^{(1)} \\
= & 2 n \pi+i m\left[\log 2 n \pi+\left(i m \log 2 n \pi+c+O\left(n^{-1} \log n\right)\right)(2 n \pi)^{-1}+O\left(\xi^{2}\right)\right] \\
& +c+\delta^{(1)} \\
= & 2 n \pi+i m \log 2 n \pi+c+(2 n \pi)^{-1}\left[-m^{2} \log 2 n \pi+i m c+i\left(c_{11}-c_{21}\right)\right] \\
& +O\left(n^{-2} \log ^{2} n\right) .
\end{aligned}
$$

Here and subsequently, the term $2 \theta$ is dropped, since its inclusion would merely lead to terms which are $O(\theta)$. We have established, for $k=1$, the following induction hypothesis:

For $p=k$, with $k$ some positive integer, let $\delta^{(p)}$ and $w^{(p)}$ be given by

$$
\begin{aligned}
& \delta^{(p)}=\sum_{j=1}^{p} n^{-i} P_{z j}(\log n)+O\left(n^{-p-1} \log ^{p} n\right) \\
& w^{(p)}=2 n \pi+i m \log 2 n \pi+c+\sum_{j=1}^{p} n^{-i} P_{w j}(\log n)+O\left(n^{-p-1} \log ^{p+1} n\right)
\end{aligned}
$$

where $P_{w j}$ and $P_{\delta j}$ are polynomials in $\log n$ of degree $j$ and less than $j$ respectively.

With the aid of TP's, iteration gives

$$
\begin{aligned}
\delta^{(k+1)} & =i \sum_{l=1}^{k+1} \frac{(-1)^{l-1}}{l}\left[\left(\epsilon_{1}^{(k)}\right)^{l}-\left(\epsilon_{2}^{(k)}\right)^{l}\right]+O\left(n^{-k-2}\right) \\
& =i \sum_{l=1}^{k+1} \frac{(-1)^{l-1}}{l}\left[\left\{\sum_{s=1}^{m_{1}} c_{1 s}\left(w^{(k)}\right)^{-s}\right\}^{l}-\left\{\sum_{s=1}^{m_{2}} c_{2 s}\left(w^{(k)}\right)^{-\cdot}\right\}^{l}\right]+O\left(n^{-k-2}\right),
\end{aligned}
$$

where, from (3.1.6) and the TP for $(1+\xi)^{-8}$,

$$
\begin{array}{rlr}
\left(w^{(k)}\right)^{-s}= & (2 n \pi)^{-\varepsilon}\left[1+\sum_{q=1}^{k+1-s}(-1)^{q} \frac{s(s+1) \cdots(s+q-1)}{q !} \xi^{q}\right] \\
& +O\left(n^{-k-2} \log ^{k+2-s} n\right) & (1 \leqq s \leqq k), \\
\left(w^{(k)}\right)^{-s}= & (2 n \pi)^{-s}[1+O(\xi)]=(2 n \pi)^{-s}+O\left(n^{-k-2} \log n\right) & (s \geqq k+1), \\
\xi= & (2 \pi)^{-1}\left[n^{-1} p_{1}+\sum_{j=1}^{k} n^{-j-1} P_{w j}\right]+O\left(n^{-k-2} \log ^{k+1} n\right), & \\
\xi^{q}= & (2 \pi)^{-q}\left[n^{-1} p_{1}+\sum_{j=1}^{k} n^{-j-1} P_{w j}\right]^{q}+o\left(n^{-k-2} \log ^{k+1} n\right) & (q \geqq 2),
\end{array}
$$

where $p_{1} \equiv i m \log n+(i m \log 2 \pi+c)$. The following lemma (whose proof is obvious, and so will be omitted) will be used:

LEMMA 3.1. If $Q=O\left(n^{-b} \log ^{a} n\right)$, with $a$ and $b$ non-negative integers, then $Q=O\left(n^{-k-2} \log ^{k+2} n\right)$ whenever $b>k+2$ (independently of $\left.a\right)$, and whenever $b=k+2, a \leqq k+2$. 
In the expression for $\xi^{g}$ preceding Lemma 3.1 , the quantity $\sigma$, given by

$$
\sigma \equiv n^{-1} p_{1}+\sum_{j=1}^{k} n^{-j-1} P_{w j}
$$

is a LC of quantities which are of the form $n^{-\beta} \log ^{\alpha} n$, where $0 \leqq \alpha<\beta \leqq k+1$, except for the first term of $n^{-1} p_{1}$, which has $\alpha=\beta=1$. Therefore, in the resulting multinomial expansion for $\sigma^{q}$, which is a LC of quantities $n^{-b} \log ^{a} n$, among those terms having the same value for $b$ one and only one will have the largest value for $a$; namely $\left(n^{-1} i m \log n\right)^{b}$, when $b=q=1,2, \cdots, k+1-s$. When $b \geqq k+2-s$, then $a \leqq k+1-s$, and $(2 n \pi)^{-8} n^{-b} \log ^{a} n=O\left(n^{-k-2} \log { }^{k+2} n\right)$ by Lemma 3.1. Therefore,

$$
\left(w^{(k)}\right)^{-s}=(2 n \pi)^{-s}+\sum_{q=1}^{k+1-s} n^{-q-s} P_{s q}(\log n)+O\left(n^{-k-2} \log ^{k+2} n\right) \quad(1 \leqq s \leqq k),
$$

where $P_{s q}$ is a polynomial of degree $q$ in $\log n$ with the leading coefficient $\left[(-1)^{q} s(s+1) \cdots(s+q-1) / q !(2 \pi)^{q+s}\right](i m)^{q}$. Similarly, since $\epsilon_{\nu}$ $=\sum_{s=1}^{m_{y}} c_{v s}\left(w^{(k)}\right)^{-s}$, it is a LC of quantities $n^{-\beta} \log ^{\alpha} n$, with $\alpha<\beta$. The multinomial expansion of its $l$ th power is a LC of quantities $n^{-b} \log ^{a} n$, with $a<b$; by Lemma 3.1 , all terms with $b \geqq k+2$ are $O\left(n^{-k-2} \log ^{k+1} n\right)$. Therefore,

$$
\delta^{(k+1)}=i \sum_{j=1}^{k+1} n^{-i} P_{\delta j}^{\prime}(\log n)+O\left(n^{-k-2} \log ^{k+1} n\right),
$$

where the polynomials $P_{\delta}^{\prime}$ are respectively of degree less than $j$. Furthermore, the analysis of $\xi^{q}$ following Lemma 3.1 applies in

$$
\log (1+\xi)=\sum_{q=1}^{k+1} \frac{(-1)^{q-1}}{q} \xi^{q}+O\left(n^{-k-2} \log ^{k+2} n\right)
$$

by merely substituting $s=0$. Therefore, $\log (1+\xi)=\sum_{q-1}^{k+1} n^{-q} P_{\xi q}(\log n)$ $+O\left(n^{-k-2} \log ^{k+2} n\right)$, where the polynomial $P_{\xi q}$ is of degree $q$ in $\log n$, with the leading term $\left[(-1)^{q-1} / q(2 \pi)^{q}\right](i m)^{q} \log ^{q} n$. By (3.1.4), (3.1.4a), and (3.1.7), this term is multiplied by $i m$, but the resulting coefficient of $\log ^{q} n$ is not affected by the addition of $\delta^{(k+1)}$ in $w^{(k+1)}=2 n \pi+i m \log w^{(k)}+c+\delta^{(k+1)}$. Therefore, even if one of $m_{1}, m_{2}$ is 0 (see the statement just above (3.1.4),

$(3.1 .8) w^{(k+1)}=2 n \pi+i m \log 2 n \pi+c+\sum_{j=1}^{k+1} n^{-j} P_{w j}^{\prime}(\log n)+O\left(n^{-k-2} \log { }^{k+2} n\right)$,

where the polynomial $P_{u j}^{\prime}=i m P_{\xi j}+i P_{\delta}^{\prime}$, and is of degree $j$ in $\log n$, with the leading coefficient $\left[(-1)^{j-1} / j(2 \pi)^{i}\right](i m)^{i+1}$. This establishes the form of (3.1.5), (3.1.6) for arbitrary integer $p>0$. From the uniqueness of the AE's in (3.1.5), (3.1.6), (3.1.7), (3.1.8) through the $k$ th term of each summation, it follows that $P_{s j}^{\prime} \equiv P_{\delta j}$, and $P_{w j}^{\prime} \equiv P_{w j}(j=1, \cdots, k)$. This completes the induction. 
CASE (2). $m_{1}=m_{2}, P_{1} \not \equiv P_{2}$. Since the term $i m \log w$ drops out of (3.1.4), $p_{1} \equiv c$, and the TP for $\log (1+\xi)$ does not appear. The essential details of Case (1) hold when all (nonzero) powers of $\log n$ are simply deleted; $p_{1}, P_{\delta}^{\prime}$ in (3.1.7), and $P_{v j}^{\prime}$ in (3.1.8) are replaced by the constants $c, c_{\delta j}$ and $c_{v o f}^{\prime}$ respectively. Therefore,

$$
w^{(p)}=2 n \pi+c+\sum_{j=1}^{p} c_{w j} n^{-j}+O\left(n^{-p-1}\right)
$$

for all integer $p>0$.

CASE (3). $P_{1} \equiv P_{2}$. Since $Q_{1}(w) \equiv Q_{2}(w)$ in (3.1.3), the result is $w=(2 n-1) \pi$ $+2 \theta$. Thus, we have proved

THEOREM 3.1. Let the ES $F(z)$ be defined by $F(z)=\sum_{\nu=1}^{N} P_{\nu}(z) e^{a_{\nu z}}(N>1)$, where the $P_{\nu}(z)$ are polynomials respectively of degree $m_{\nu}$ in the complex variable $z$, and the $a_{\nu}$ are distinct complex constants. Let the consecutive vertices $a_{1}^{*}$ and $a_{2}^{*}$ of the ID for $F(z)$ be the only EP's on the side joining them, and ordered so that $\phi_{w}=\pi / 2+\arg \left(a_{1}^{*}-a_{2}^{*}\right)$, where $\phi_{w}$ is the argument of the outward normal to this side. The zeros $z_{n}$ corresponding to this side, w.f.m.p.e., are simple, and given by

$$
\begin{aligned}
z_{n}=\left(e^{i \phi_{w}} /\left|a_{1}-a_{2}\right|\right)[2 n \pi & +i m \log \left(2 n \pi /\left|a_{1}-a_{2}\right|\right)+c \\
& \left.+\sum_{j=1}^{k} n^{-i} p_{j}(\log n)+O\left(n^{-k-1} \log ^{k+1} n\right)\right],
\end{aligned}
$$

where $k$ is an arbitrary positive integer, $m=m_{1}-m_{2}, c=-\pi-m \phi_{w}$ $+i \log \left(C_{10} / C_{20}\right), C_{10}$ and $C_{20}$ are the leading coefficients of $P_{1}$ and $P_{2}$ respectively, and $p_{j}(\log n)$ is a polynomial, of degree $j$ in $\log n$ with the leading coefficient $(2 \pi / j)(m / 2 \pi i)^{j+1}$, reducing identically to a constant when $m_{1}=m_{2}$ and to zero when $P_{1} \equiv P_{2}$.

In addition to Theorem 3.1, the following results will characterize $\sigma\left(T_{3}^{-}\right)$, the spectrum of $T_{3}^{-}(D)$.

TheOREM 3.2. If $\Delta(\rho)$, with $\rho^{n}=\lambda$, is the CD of one of the $E E^{\prime} s-d^{n} y / d x^{n}$ $=\lambda y$ and $d^{n} y / d x^{n}=\lambda y$, and $\omega_{1} \equiv e^{i \pi / n}$, then $\Delta\left(\rho \omega_{1}^{2}\right)=(-1)^{n-1} \Delta(\rho)$.

Proof. By Corollary 2.1, it is sufficient to consider $\Delta(\rho)=\operatorname{det} U_{i}\left(y_{j}\right)$ as formed from the respective FS's $y_{j}=e^{\rho \omega_{j} x}$ and $y_{j}=e^{\rho \nu_{j} x}$, where $\omega_{j}=e^{i(2 j-1) x / n}$ and $\nu_{j}=e^{i 2 j \pi / n}(j=1, \cdots, n)$. Replacing $\rho$ by $\rho \omega_{1}^{2}$ merely shifts the last column of det $U_{i}\left(y_{j}\right)$ to the position of the first, and is therefore equivalent to applying $n-1$ successive transpositions of two adjacent columns.

CoRollary 3.2.1. If $\rho_{0}$ is a zero of $\Delta(\rho)$ for $T_{3}^{-}(D)$, then $\rho_{0} \omega_{1}^{2 l}(l=1, \cdots, n-1)$ are zeros of $\Delta(\rho)$.

Proof. This follows from $l$ successive applications of Theorem 3.2. 
Apart from requiring an additional condition in a subcase of Case 4 , the BC's I (B) for $T_{3}^{-}(D)$ are nondegenerate, since using their canonical forms in (2.6.1) results in $P_{a}$ and $P_{b}$ of unequal degrees $m_{a}$ and $m_{b}$ respectively. In the (a) cases $m_{a}>m_{b}$, and the respective values of $m_{a}$ are as follows:

\begin{tabular}{c|ccccccc} 
Case & $1(\mathrm{a})$ & $2(\mathrm{a})$ & $3(\mathrm{a})$ & $4(\mathrm{a})$ & $5(\mathrm{a})$ & $6(\mathrm{a})$ & $7(\mathrm{a})$ \\
\hline$m_{a}=$ & 5 & 4 & 4 & $3,2,1$ & 2 & 2 & 1
\end{tabular}

In the corresponding Cases $1(\mathrm{~b})-7(\mathrm{~b}), m_{b}>m_{a}$ and the values listed above for $m_{a}$ become those for $m_{b}$. It is unnecessary to consider these cases separately. The significant items for the spectrum of $T_{3}^{-}(D)$ are exhibited below. The AR's including the first two listed values of $\phi_{w}$ are the only ones for which Theorem 3.1 will be applied; by applying the substitution $\rho=-\rho^{\prime}$ and Corollary 3.2.1, the AE's for the remaining AR's will then result.

\begin{tabular}{ccccccccc}
\hline \hline Case & $P_{a}$ & $P_{b}$ & ID & Vertices & $a_{1}$ & $a_{2}$ & $\left|a_{1}-a_{2}\right|$ & $\phi_{w 0}$ \\
\hline$(1)$ & $\neq 0$ & $\not \equiv 0$ & regular hexagon & $\pm \omega_{1}^{*}, \pm \omega_{2}^{*}, \pm \omega_{3}^{*}$ & $\omega_{1}$ & $-\omega_{2}$ & 1 & $-\pi / 6$ \\
$(2)$ & $\neq 0$ & $\equiv 0$ & equilateral triangle & $\omega_{1}{ }^{*}, \omega_{2}^{*}, \omega_{3}^{*}$ & $\omega_{1}$ & $\omega_{3}$ & $3^{1 / 2}$ & 0 \\
$(3)$ & $\equiv 0$ & $\neq 00$ & equilateral triangle & $-\omega_{1}^{*},-\omega_{2}^{*},-\omega_{3}^{*}$ & $-\omega_{1}$ & $-\omega_{3}$ & $3^{1 / 2}$ & $\pi$ \\
\hline
\end{tabular}

REMARK 3.3.1. In all three cases, the term $C \rho^{3}$ in (2.6.1) does not affect the asymptotic character of the zeros, since the origin, which is an EP only when $C \neq 0$, lies inside the ID.

REMARK 3.3.2. Under the convention $-\pi<\arg z \leqq \pi$, used here and subsequently, $\left|e^{z}\right|$ increases, for a given $|z|$, as $|\arg z|$ decreases, since $\left|e^{z}\right|$ $=e^{|z| \cos \arg z}$.

CASE (1). By Theorem 3.1, and the fact that the polynomials in (2.5.1) have the arguments $\rho \omega_{l}$, the $O(1)$ term is $-\pi-\left(m_{a}-m_{b}\right)(\pi / 6)$ $+i \log \left(C_{a 0} \omega_{1}^{m_{a}} / C_{b 0} \omega_{2}^{{ }^{m b}}\right)$. Therefore, $\rho_{n}=w_{n} e^{-i \pi / 6}$, where

$$
\begin{array}{r}
w_{n}=\left[2 n \pi+i\left(m_{a}-m_{b}\right) \log 2 n \pi-\pi-\left(m_{a}+7 m_{b}\right) \frac{\pi}{6}+i \log \left(C_{a 0} / C_{b 0}\right)\right. \\
\left.+\sum_{j=1}^{k} n^{-i} p_{j}(\log n)+O\left(n^{-k-1} \log ^{k+1} n\right)\right] .
\end{array}
$$

When (2.6.1) is written as

$$
3 \rho^{3} \Delta(\rho)=C \rho^{3}+\sum_{s=0}^{m_{a}} C_{a s} \sum_{l=1}^{3}\left(\rho \omega_{l}\right)^{m_{a}-s} e^{\rho \omega_{l}}+\sum_{s=0}^{m_{b}} C_{b_{s}} \sum_{l=1}^{3}\left(\rho \omega_{l}\right)^{m_{b}-s} e^{-\rho \omega_{l}},
$$

the substitution $\rho=-\rho^{\prime}$ transforms its right-hand member into an expression of the same form, but with $C_{a s}^{\prime}=(-1)^{m_{b}-s} C_{b s}, C_{b s}^{\prime}=(-1)^{m_{a}-s} C_{a s}, P_{a}^{\prime}$ of degree $m_{a}^{\prime}=m_{b}, P_{b}^{\prime}$ of degree $m_{b}^{\prime}=m_{a}$. Application of (3.3.1) gives $\rho_{n}^{\prime}=w_{n}^{\prime} e^{-i \pi / 6}$, where 


$$
\begin{array}{r}
w_{n}^{\prime}=\left[2 n \pi+i\left(m_{b}-m_{a}\right) \log 2 n \pi-\pi-\left(m_{b}+7 m_{a}\right) \frac{\pi}{6}+i \log \left(C_{a 0}^{\prime} / C_{b 0}^{\prime}\right)\right. \\
\left.+\sum_{j=1}^{k} n^{-i} p_{j}^{\prime}(\log n)+O\left(n^{-k-1} \log { }^{k+1} n\right)\right],
\end{array}
$$

where the primed items other than $w_{n}^{\prime}$ are obtained from the corresponding unprimed ones in (3.3.1) by means of the substitutions following (3.3.2). Since $\rho=\rho^{\prime} e^{ \pm i \pi}$, by Corollary 3.2.1 we have the following result for Case (1):

The zeros of $\Delta(\rho)$ for $T_{3}^{-}(D)$, w.f.m.p.e., are simple, and given by $w_{n} e^{-i b \pi / 6}$, $w_{n} e^{-i \pi / 6}, w_{n} e^{i \pi / 2}, w_{n}^{\prime} e^{-i \pi / 2}, w_{n}^{\prime} e^{i \pi / 6}, w_{n}^{\prime} e^{i 5 \pi / 6}$, with $w_{n}$ and $w_{n}^{\prime}$ given by (3.3.2) and (3.3.3) respectively.

CASE (2). By Theorem 3.1, Case (2), and Corollary 3.2.1, we have the following result for the present Case (2): The zeros of $\Delta(\rho)$ for $T_{3}(D)$, w.f.m.p.e., are simple, and given by $w_{n} e^{-i 2 \pi / 3} / 3^{1 / 2}, w_{n} / 3^{1 / 2}$, and $w_{n} e^{i 2 \pi / 8} / 3^{1 / 2}$, where

$$
w_{n}=\left[(2 n-1) \pi-\frac{2 \pi m_{a}}{3}+\sum_{j=1}^{k} c_{j} n^{-j}+O\left(n^{-k-1}\right)\right],
$$

and the $c_{j}$ are constants.

CASE (3). By the substitution $\rho=-\rho^{\prime}$, we have Case (2) again, but with $C_{a s}^{\prime}=(-1)^{m_{b}-s} C_{b s}$, and $P_{a}^{\prime}$ of degree $m_{a}^{\prime}=m_{b}$. Therefore, we have the following result for Case (3): The zeros of $\Delta(\rho)$ for $T_{3}^{-}(D)$, w.f.m.p.e., are simple, and given by $w_{n}^{\prime} e^{-i \pi / 3} / 3^{1 / 2}, w_{n}^{\prime} e^{i \pi / 3} / 3^{1 / 2}$, and $w_{n}^{\prime} e^{i \pi} / 3^{1 / 2}$, where

$$
w_{n}^{\prime}=\left[(2 n-1) \pi-\frac{2 \pi m_{b}}{3}+\sum_{j=1}^{k} c_{j}^{\prime} n^{-j}+O\left(n^{-k-1}\right)\right],
$$

and the $c_{j}^{\prime}$ are constants.

The remaining analysis will not require these AE's, but the knowledge that for $\phi=\arg \rho$ bounded away from $\phi_{w}$ and for $r=|\rho|$ sufficiently large, the corresponding $\lambda$ is not in the spectrum of $T$. For example, examination of these AE's shows that $\lambda \notin \sigma\left(T_{3}^{-}\right)$for $\epsilon<\phi<\pi / 6-\epsilon$, as $r \rightarrow \infty$. This fact will be used in the next section.

4. Completeness of the eigenfunctions of $T_{3}^{-}(D)$ in $L_{2}(0,1)$. If $\lambda \notin \sigma\left(T_{n}\right)$, then $G(x ; t, \lambda)$ is the kernel of the integral operator [IO] which is called the resolvent operator of $T_{n}\left[R_{\lambda}\right]$. In $L_{2}(0,1)$, the norm of $R_{\lambda}\left[\left\|R_{\lambda}\right\|\right]$ satisfies

$$
\left\|R_{\lambda}\right\| \leqq \sup _{0 \leqq x \leqq 1}\left[\int_{0}^{1}|G(x ; t, \lambda)|^{2} d t\right]^{1 / 2}
$$

The notations $G(x ; t, \lambda)$ and $G(x ; t, \rho)$ will be used interchangeably. It will be proved that, with a possible exception to be specified later, the eigenfunctions of $T_{3}^{-}(D)$ span $L_{2}(0,1)$, provided that the BC's $(0.2)$ are nondegenerate. This will be done by applying 
TheOREM 4.0 (DUNFord-Schwartz) [5]. Let $T$ be an unbounded operator on $L_{2}(0,1)$ whose resolvent $R_{\lambda}$ is an IO with kernel $G(x ; t, \lambda)$ such that $\int_{0}^{1} \int_{0}^{1}|G(x ; t, \lambda)|^{2} d x d t<\infty$. If for some $\lambda \notin \sigma(T)$ and lying outside of disjoint $A R$ 's with a common vertex, each of angle $<\pi / 2$ but such that the sum of their angles is arbitrarily close to $2 \pi$, we have $\left\|R_{\lambda}\right\|<K|\lambda|{ }^{k}$, where $K, k$ are integers with the same respective values for all such $\lambda$, then the eigenfunctions of $T$ span $L_{2}(0,1)$.

We proceed to show that the hypotheses of Theorem 4.0 are satisfied by $R_{\lambda}$ of $T_{3}^{-}(D) . G(x ; t, \lambda)$ is continuous in $x$ and $t$ over the unit square defined by $0 \leqq x \leqq 1,0 \leqq t \leqq 1$; therefore, $\int_{0}^{1} \int_{0}^{1}|G|^{2} d x d t<\infty$ for all $\lambda \notin \sigma\left(T_{3}^{-}\right)$. By the results of the preceding section (or Schwengeler's work), the zeros of $\Delta(\rho)$ approach, in an angular sense, the $\phi_{w}$ rays for which $\phi_{w}$ is an odd multiple of $\pi / 6$ in Case (1) and an even multiple in Cases (2) and (3). More precisely, these zeros, w.f.m.p.e., lie in the AR pairs defined by $\phi_{w}-\epsilon<\phi<\phi_{w}$ and $\phi_{w}<\phi$ $<\phi_{w}+\epsilon$, where $\epsilon>0$ and suitably small, and $\phi_{w}=s \pi / 6(s= \pm 1, \cdots, \pm 6)$. Therefore, $\lambda \notin \sigma\left(T_{3}^{-}\right)$as $r \rightarrow \infty$ when $(s-1) \pi / 6+\epsilon<\phi<s \pi / 6-\epsilon$; that is, when $(s-1) \pi / 2+3 \epsilon<\arg \lambda<s \pi / 2-3 \epsilon(s=-5,-4, \cdots, 6)$. Therefore, $\lambda \notin \sigma\left(T_{3}^{-}\right)$, if we impose the restrictions $(s-1) \pi / 6+\epsilon<\phi<(s-1) \pi / 6+\left(\epsilon+\epsilon^{\prime}\right)$, and $s \pi / 6-\left(\epsilon+\epsilon^{\prime}\right)<\phi<s \pi / 6-\epsilon$, where $\epsilon^{\prime}>0$ and arbitrarily small. The corresponding $\lambda$ lie outside the disjoint AR's, in the $\lambda$-plane, given by $(s-1) \pi / 2$ $+3\left(\epsilon+\epsilon^{\prime}\right)<\arg \lambda<s \pi / 2-3\left(\epsilon+\epsilon^{\prime}\right)$ and any four consecutive integer values of $s$; we select $s=-1,0,1,2$. These disjoint AR's obviously meet the requirements of Theorem 4.0. For the remaining values of $s$ these AR pairs are merely duplicated. Estimates are needed for $\left[\int_{0}^{1}|Z(x ; t, \lambda)|^{2} d t\right]^{1 / 2}$ and for $\Delta$, in accordance with (4.0.1). We designate the four AR pairs in the $\rho$-plane corresponding to $s=1,0,2,-1$ as AR pairs (1), (2), (3), (4) respectively. It will be seen later that the remaining values of $s$ result in the same estimates as these do. We require the arrangement in Table 4.2.1 of the exponentials $\exp \left( \pm \rho \omega_{l} q\right)$, for $q$ real and $\rho$ in the AR pairs (1)-(4) respectively, in descending order of absolute value as $r \rightarrow \infty$. For $q>0$, the first three exponentials $\rightarrow \infty$ in absolute value and the last three $\rightarrow 0$. For $q<0$, the descending order is reversed, and the preceding statement then applies.

TABLE 4.2 .1

\begin{tabular}{cc}
\hline AR Pair & Exponentials in descending order for $q>0$ \\
\hline$(1)$ & $e^{-\rho \omega_{2} q}, e^{\rho \omega_{2} q}, e^{\rho \omega_{1} q}, e^{-\rho \omega_{1} q}, e^{-\rho \omega_{3} q}, e^{\rho \omega_{2} q} ;$ \\
$(2)$ & $e^{-\rho \omega_{2} q}, e^{\rho \omega_{1} q}, e^{\rho \omega_{3} q}, e^{-\rho \omega_{3} q}, e^{-\rho \omega_{1} q}, e^{\rho \omega_{2} q} ;$ \\
$(3)$ & $e^{\rho \omega_{3} q}, e^{-\rho \omega_{2} q}, e^{-\rho \omega_{1} q}, e^{\rho \omega_{1} q}, e^{\rho \omega_{2} q}, e^{-\rho \omega_{8} q} ;$ \\
$(4)$ & $e^{\rho \omega_{1} q}, e^{-\rho \omega_{2} q}, e^{-\rho \omega_{3} q}, e^{\rho \omega_{8} q}, e^{\rho \omega_{2} q}, e^{-\rho \omega_{1} q} ;$ \\
\hline
\end{tabular}

The estimating of $\left[\int_{0}^{1}|Z(x ; t, \lambda)|^{2}\right]^{1 / 2}$ will be simplified by noting that 


$$
\int_{0}^{1}|Z|^{2} d t=\int_{0}^{1}\left|\sum_{k} C_{k} M_{k}\right|^{2} d t \leqq \int_{0}^{1}\left(\sum_{k}\left|C_{k} M_{k}\right|\right)^{2} d t .
$$

By the Schwarz inequality,

$$
\left(\int_{0}^{1}\left|C_{\kappa_{i}} M_{\alpha_{i}}\right|\left|C_{k_{j}} M_{\alpha_{j}}\right| d t\right)^{2} \leqq \int_{0}^{1}\left|C_{\kappa_{j}} M_{\alpha_{i}}\right|^{2} d t \int_{0}^{1}\left|C_{k_{j}} M_{k_{j}}\right|^{2} d t,
$$

and so the mixed product terms in the expansion of $\int_{0}^{1}\left(\sum_{k}\left|C_{k} M_{k}\right|\right)^{2} d t$ are not of larger order than the larger of $\int_{0}^{1}\left|C_{k_{i}} M_{k_{i}}\right|{ }^{2} d t$ and $\int_{0}^{1}\left|C_{k_{j}} M_{k_{j}}\right|^{2} d t$. Therefore,

$$
\int_{0}^{1}|Z|^{2} d t=O\left[\int_{0}^{1} \sum_{\kappa}\left|C_{k} M_{k}\right|^{2} d t\right]=O\left[\max _{C_{k} \neq 0} \int_{0}^{1}\left|M_{\kappa}\right|^{2} d t\right],
$$

where the last item in brackets means the $\int_{0}^{1}\left|M_{k}\right|{ }^{2} d t$ of largest order over all $\kappa \ni C_{k} \neq 0$. Furthermore, $O\left[\int_{0}^{1}\left|M_{k}\right|^{2} d t\right]$ is the larger of $O\left[\int_{(x>t)}\left|M_{k}\right|^{2} d t\right]$ and $O\left[\int_{(x<t)}\left|M_{k}\right|^{2} d t\right]$. Also, the factor $\left|\rho^{m_{k}}\right|^{2}$ of $\left|M_{k}\right|^{2}$ will be regarded as written outside the integral sign and accounted for after integrating the estimates for $\left|\rho^{-m_{k}} M_{k}\right|^{2}$. These integrals may (and will) be used instead of the $\int_{0}^{1}\left|\rho^{-m_{k} M_{k}}\right|^{2} d t$ themselves, since the $O$ and $\int$ symbols may be written in reverse order. For brevity, the transitive relation $p=O[q]$ will be designated by $p<\sim q$, and $\left|\rho^{-m_{k}} M_{k}\right|$ by $Q_{k}$. We shall use the ordering of the $M_{k}$ in Table 2.5.1. Unless otherwise specified, the upper sign of a \pm or $\mp$ symbol will refer to the AR pairs (1) and (3), and the lower to the AR pairs (2) and (4). fore,

$\kappa=1$. For $x>t, Q_{1}<\sim \exp \{(x-t) r \cos (\pi / 3 \mp \phi)\}$, by Table 4.2.1. There-

$$
\int_{0}^{1} Q_{1}^{2} d t=\int_{0}^{x} Q_{1}^{2} d t<\sim \frac{1}{r} \exp \{2 r \cos (\pi / 3 \mp \phi)\} .
$$

$\kappa=2, \cdots, 10$. For $x>t, Q_{\alpha}=\left|u_{\alpha}(\rho(1-t)) u_{\beta}(\rho x)-u_{3}(\rho(x-t)) u_{\gamma}(\rho)\right|$, where $\alpha+\beta=3+\gamma$, by (2.5.02). We drop the $e^{z \omega_{2}}$ term of (2.5.01) for each of these $u$ 's, since $e^{\rho \omega_{2} q}=e^{-\rho q} \rightarrow 0$ for $q>0$ in all four AR pairs, by Table 4.2.1. On using (2.5.08) and applying the identity

$$
\cos A \cos B \equiv[\cos (A+B)+\cos (A-B)] / 2,
$$

two of the resulting cosines cancel out, giving

$$
\begin{aligned}
Q_{k}<\sim\left|e^{(x-t+1)_{\rho} / 2}\right| \mid \cos [ & \left.(\beta-\alpha) \frac{\pi}{3}+\frac{3^{1 / 2}}{2} \rho(1-t-x)\right] \\
& \quad-\cos \left[(\gamma-3) \frac{\pi}{3}+\frac{3^{1 / 2}}{2} \rho(x-t-1)\right] \mid
\end{aligned}
$$

Since $O[|\cos (\theta+z)|]=O[|\sin (\theta+z)|]=O\left[e^{|\operatorname{Im} z|}\right]=O\left[e^{|z| \mid \sin \text { arg } z \mid}\right]$ for $\theta$ real, 
the first cosine in (4.4.2) is not of greater order than the second, and so is dropped. Therefore,

$$
\begin{aligned}
Q_{k}<\sim & \exp \{(1 / 2)(x-t+1) r \cos \phi\} \\
& \cdot \exp \left\{\left(3^{1 / 2} / 2\right)(1+t-x) r( \pm \sin \phi)\right\}
\end{aligned}
$$

The cosine addition formula gives

$$
Q_{\kappa}<\sim \exp \{r \cos (\pi / 3 \mp \phi)\} \exp \{(x-t) r \cos (\pi / 3 \pm \phi)\} .
$$

Replacing $x$ by the value 1 gives

$$
Q_{\kappa}<\sim \exp \{r \cos \phi\} \exp \{-\operatorname{tr} \cos (\pi / 3 \pm \phi)\},
$$

and integration results in

$$
\int_{0}^{x} Q_{k}^{2} d t<\sim \frac{1}{r} e^{2 r \cos \phi}
$$

For $x<t$, keeping only the exponential of largest order for each factor gives

$$
\begin{aligned}
Q_{\star} & <\sim \exp \{(1+x-t) r \cos (\pi / 3 \mp \phi)\}, \\
\int_{x}^{1} Q_{x}^{2} d t & <\sim \frac{1}{r} \exp \{2 r \cos (\pi / 3 \mp \phi)\} .
\end{aligned}
$$

$\kappa=11, \cdots, 19$. For $x>t$, the calculation is similar to the last one, but now the exponential $e^{\rho \omega_{2} q}$ is kept. Thus, $Q_{\kappa}<\sim e^{(1-x+t) r \cos \phi}$, and

$$
\int_{0}^{x} Q_{k}^{2} d t<\sim \frac{1}{r} e^{2 r \cos \phi} \text {. }
$$

For $x<t, Q_{\alpha}=\left|u_{\alpha}(-\rho t) u_{\beta}(\rho(x-1))-u_{3}(\rho(x-t)) u_{\gamma}(-\rho)\right|$, with $\alpha+\beta=3+\gamma$. Here $q<0$, and so, by Table 4.2.1, we drop the $\exp \left(\rho e^{\mp i \pi / 8} q\right)$ term. Applying (3.1.1) to the remaining two terms of (2.5.01), we then proceed as for $Q_{\kappa}(\kappa=2, \cdots, 10)$ for $x>t$. Thus,

$$
Q_{\kappa}<\sim e^{r \cos \phi} \exp \{(t-x) r \cos (2 \pi / 3 \mp \phi)\} \text {. }
$$

Now, $\cos (2 \pi / 3-\phi)$ is negative for the AR pair (1) and positive for the AR pair (3), and $\cos (2 \pi / 3+\phi)$ is negative for the AR pair (2) and positive for the AR pair (4). For $\cos (2 \pi / 3 \mp \phi)<0$, integration gives

$$
\int_{x}^{1} Q_{k}^{2} d t<\sim \frac{1}{r} e^{2 r \cos \phi} \text { (in the AR pairs (1) and (2)), }
$$

and for $\cos (2 \pi / 3 \mp \phi)>0$, integrating and replacing $x$ by the value 0 give 


$$
\int_{x}^{1} Q_{x}^{2} d t<\sim \frac{1}{r} \exp \{2 r \cos (\pi / 3 \mp \phi)\},
$$

in which the upper and lower signs apply to the AR pairs (3) and (4) respectively.

$\kappa=20$. For $x<t, Q_{k}<\sim e^{(t-x) r \cos \phi}$, and

$$
\int_{0}^{1} Q_{x}^{2} d t=\int_{x}^{1} Q_{x}^{2} d t<\sim \frac{1}{r} e^{2 r \cos \phi} .
$$

By allowing for the ranges of $\phi$ in the respective AR pairs, by keeping only the larger of the respective estimates for $\int_{0}^{x} Q_{x}^{2} d t$ and $\int_{x}^{1} Q_{x}^{2} d t$, and noting that $\left[\int_{0}^{1}\left|M_{k}\right|^{2} d t\right]^{1 / 2}$ has the factor $r^{m_{k}-1 / 2}$, we obtain

TABLE 4.5.1

\begin{tabular}{|c|c|c|c|c|}
\hline & 1 & $2, \cdots, 10$ & $11, \cdots, 19$ & 20 \\
\hline (1) & $O\left[e^{r \cos (\pi / 3-\phi)}\right]$ & $O\left[e^{r \cos \phi}\right]$ & $O\left[e^{r \cos \phi}\right]$ & $O\left[e^{r \cos \phi}\right]$ \\
\hline (2) & $O\left[e^{r \cos (\pi / 3+\phi)}\right]$ & $O\left[e^{r \cos \phi}\right]$ & $O\left[e^{r \cos \phi}\right]$ & $O\left[e^{r \cos \phi}\right]$ \\
\hline (3) & $O\left[e^{r \cos (\pi / 8-\phi)}\right]$ & $O\left[e^{r \cos (\pi / 3-\phi)}\right]$ & $O\left[e^{r \cos (\pi / 3-\phi)}\right]$ & $O\left[e^{r \cos \phi} \phi\right]$ \\
\hline (4) & $O\left[e^{r \cos (\pi / 3+\phi)}\right]$ & $O\left[e^{r \cos (\pi / 3+\phi)}\right]$ & $O\left[e^{r \cos (\pi / 3+\phi)}\right]$ & $O\left[e^{r \cos \phi}\right]$ \\
\hline
\end{tabular}

Estimates for $r^{-m_{k}+1 / 2}\left[\int_{0}^{1}\left|M_{\kappa}\right|^{2} d t\right]^{1 / 2}$

By (2.5.1) and (2.6.1),

$$
\begin{gathered}
\Delta(\rho)=\sum_{k=1}^{20} C_{k} \mu_{k}, \quad \text { where } \sum_{k=1,20} C_{k} \mu_{k}=C / 3, \\
\sum_{k=2}^{10} C_{k} \mu_{k}=\frac{1}{3} \rho^{-3} \sum_{l=1}^{3} P_{a}\left(\rho \omega_{l}\right) e^{\rho \omega l}, \quad \sum_{k=11}^{10} C_{k} \mu_{k}=\frac{1}{3} \rho^{-8} \sum_{l=1}^{8} P_{b}\left(\rho \omega_{l}\right) e^{-\rho \omega l} .
\end{gathered}
$$

Since we have excluded the degenerate case $P_{a} \equiv 0, P_{b} \equiv 0$, we now consider the following three cases: Case (1) $P_{a} \neq 0, P_{b} \neq 0$; Case (2) $P_{a} \not \equiv 0, P_{b} \equiv 0$; Case (3) $P_{a} \equiv 0, P_{b} \neq 0$. In all three cases, lower bounds are obtained by applying the triangle inequality and factoring out the leading term $Q$ of the PT of $\Delta(\rho)$, in accordance with Table 4.2.1. Thus, $|\Delta|=|Q+(\Delta-Q)|$ $\geqq|Q|-|\Delta-Q|=|Q|(1-|(\Delta-Q) / Q|)=|Q|(1-h)$, where $0 \leqq h$ $=|(\Delta-Q) / Q|=O\left(r^{-1}\right)$.

CASE (1). $P_{a} \not \equiv 0, P_{b} \not \equiv 0$. We have

$$
|\Delta| \geqq\left|\frac{1}{3} \rho^{-3} C_{b 0}\left(\rho \omega_{2}\right)^{m_{b}} e^{-\rho \omega_{2}}\right|(1-h)=\frac{1}{3}\left|C_{b 0}\right| r^{m b-3} e^{r \cos \phi}(1-h),
$$

where $h=h_{1}, h_{2}$ for the AR pairs (1) and (2) respectively; 


$$
\begin{aligned}
|\Delta| & \geqq\left|(1 / 3) \rho^{-3} C_{a 0}\left(\rho \omega_{3}\right)^{m_{a}} e^{\rho \omega_{3}}\right|\left(1-h_{3}\right) \\
& =(1 / 3)\left|C_{a 0}\right| r^{m_{a}-3} e^{r \cos (\pi / 3-\phi)}\left(1-h_{8}\right)(\mathrm{AR} \text { pair }(3)) ; \\
|\Delta| & \geqq\left|(1 / 3) \rho^{-3} C_{a 0}\left(\rho \omega_{1}\right)^{m_{a}} e^{\rho \omega_{1}}\right|\left(1-h_{4}\right) \\
& =(1 / 3)\left|C_{a 0}\right| r^{m_{a}-3} e^{r \cos (\pi / 3+\phi)}\left(1-h_{4}\right) \text { (AR pair (4)). }
\end{aligned}
$$

For all $r$ sufficiently large, there is a positive constant $K_{\Delta}$ smaller than any of the four positive quantities $(1 / 3)\left|C_{b 0}\right|(1-h)\left(h=h_{1}, h_{2}\right)$, $(1 / 3)\left|C_{a 0}\right|\left(1-h_{3}\right),(1 / 3)\left|C_{a 0}\right|\left(1-h_{4}\right)$. Let $k_{\Delta}$ be the smaller of the unequal (by §2) quantities $m_{a}-3, m_{b}-3$. Then $|\Delta|>K_{\Delta} r^{k} \Delta e^{r \cos \phi}$ (AR pairs (1), (2)), $|\Delta|>K_{\Delta} r^{k} e^{r \cos \left(\pi / 3 \mp_{\phi}\right)}$ (AR pairs (3), (4) respectively). The largest entries in Table 4.5.1 are $O\left[e^{r \cos \phi}\right], O\left[e^{r \cos \phi}\right], O\left[e^{r \cos (x / 3-\phi)}\right], O\left[e^{r \cos (\pi / 3+\phi)}\right]$ for the AR pairs (1), (2), (3), (4) respectively. By our exclusion of degenerate cases, not all of the $C_{k}$ vanish for $\kappa=2, \cdots, 19$. By (4.3.2),

$$
\left(\int_{0}^{1}|Z|^{2} d t\right)^{1 / 2}=O\left[\max _{C_{k} \neq 0}\left(\int_{0}^{1}\left|M_{\kappa}\right|^{2} d t\right)^{1 / 2}\right],
$$

and $\left[\int_{0}^{1}\left|M_{k}\right|{ }^{2} d t\right]^{1 / 2}$ equals $r^{m_{k}-1 / 2}$ times the corresponding entry in Table 4.5.1. Let $k_{Z}$ be the largest value of the $m_{k}-1 / 2 \quad(\kappa=1, \cdots, 20)$ such that $C_{k} \neq 0$. Then for all $r$ sufficiently large, there are positive constants $K_{1}, K_{2}$, $K_{3}, K_{4}$ such that $\left(\int_{0}^{1}|Z|^{2} d t\right)^{1 / 2}<K_{1} r^{k} Z e^{r \cos \phi}, K_{2} r^{k} z e^{r \cos \phi}, K_{3} r^{k} z e^{r \cos (\pi / 3-\phi)}$, $K_{4} r^{k} z e^{r \cos (\pi / 3+\phi)}$ for the AR pairs (1), (2), (3), (4) respectively. Let $K_{Z}$ be the largest of the constants $K_{1}, K_{2}, K_{3}$, and $K_{4}$. Then for the AR pairs (1), (2), (3), (4), $\left(\int_{0}^{1}|Z|^{2} d t\right)^{1 / 2}<K_{Z} r^{m} Z$ times $e^{r \cos \phi}, e^{r \cos \phi}, e^{r \cos (\pi / 3-\phi)}, e^{r \cos (\pi / 3+\phi)}$

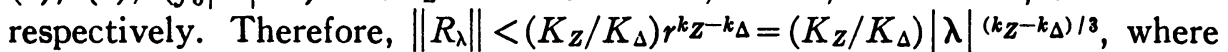
the constants $\left(K_{Z} / K_{\Delta}\right)$ and $\left(k_{Z}-k_{\Delta}\right) / 3$ are independent of the choice of $\lambda$ in the AR pairs (1)-(4) inclusive, for all sufficiently large $r$. There are integers $K, k \ni K \geqq K_{Z} / K_{\Delta}, k \geqq\left(k_{Z}-k_{\Delta}\right) / 3$. This completes the proof in Case (1).

CASE (2). $P_{a} \not \equiv 0, P_{b} \equiv 0$. For the AR pair (1),

$$
|\Delta| \geqq\left|\frac{1}{3} \rho^{-3} C_{a 0}\left(\rho \omega_{3}\right)^{m_{a}} e^{\rho \omega_{8}}\right|\left(1-h_{1}^{\prime}\right)=\frac{1}{3}\left|C_{a 0}\right| r^{m_{a}-3} e^{r \cos (\pi / 3-\phi)}\left(1-h_{1}^{\prime}\right) .
$$

We see at once from Table 4.5.1 that $\left(\int_{0}^{1}|Z|^{2} d t\right)^{1 / 2}$ is possibly of exponentially larger order than $\Delta$ unless $C_{k}=0(\kappa=2, \cdots, 20)$, which is a degenerate case. Therefore, Theorem 4.0 is inapplicable.

CAse (3). $P_{a} \equiv 0, P_{b} \neq 0$. For the AR pair (3),

$$
|\Delta| \geqq\left|\frac{1}{3} \rho^{-3} C_{b 0}\left(\rho \omega_{2}\right)^{m b} e^{-\rho \omega_{2}}\right|\left(1-h_{3}^{\prime}\right)=\frac{1}{3}\left|C_{b 0}\right| r^{m_{b}-3} e^{r \cos \phi}\left(1-h_{3}^{\prime}\right) .
$$

By Table 4.5.1, $\left(\int_{0}^{1}|Z|^{2} d t\right)^{1 / 2}$ is possibly of exponentially larger order than $\Delta$ 
unless $C_{\kappa}=0(\kappa=1, \cdots, 19)$, which is a degenerate case. Therefore, Theorem 4.0 is inapplicable.

REMARK. The estimates in this proof remain the same if four consecutive values of $s$ other than $-1,0,1,2$ are used, in view of (2.5.07). Also, the possible exception referred to earlier occurs when $P_{a}\left(\rho \omega_{l}\right)$ or $P_{b}\left(\rho \omega_{l}\right)$ is identically zero. Thus, we have proved

TheOREM 4.5. If $P_{a}\left(\rho \omega_{l}\right)$ and $P_{b}\left(\rho \omega_{l}\right)$, as given by (2.6.1), are both not identically zero, then the eigenfunctions of $T_{3}^{-}(D)$ span $L_{2}(0,1)$.

\section{BIBLIOGRAPHY}

1. G. D. Birkhoff, On the asymptotic character of the solutions of certain linear differential equations containing a parameter, Trans. Amer. Math. Soc. vol. 9 (1908) pp. 219-231.

2. - Boundary-value and expansion problems of ordinary linear differential equations, Trans. Amer. Math. Soc. vol. 9 (1908) pp. 373-395.

3. E. A. Coddington and N. Levinson, Theory of ordinary differential equations, New York, McGraw-Hill Book Co., Inc., 1955.

4. R. Courant and D. Hilbert, Methods of mathematical physics, vol. 1, New York, Interscience Publishers, Inc., 1953.

5. N. Dunford and J. T. Schwartz, Linear operators. Part II, New York, Interscience Publishers, Inc. (forthcoming).

6. A. Erdélyi, W. Magnus, F. Oberhettinger and F. G. Tricomi, Higher transcendental functions, vol. 3, New York, McGraw-Hill Book Co., Inc., 1955, pp. 212-217.

7. S. Hoffman, Second order linear differential operators defined by irregular boundary conditions, Ph.D. dissertation, Yale, 1957.

8. J. W. Hopkins, Some convergent developments associated with irregular boundary conditions, Trans. Amer. Math. Soc. vol. 20 (1919), pp. 249-259.

9. E. L. Ince, Ordinary differential equations, New York, Dover Publications, Inc., 1956.

10. T. Muir, A treatise on the theory of determinants, New York, Dover Publications, Inc., 1960, pp. 213-216.

11. F. Riesz and B. Sz-Nagy, Functional analysis, New York, Ungar Publishing Co., 1955, pp. $145-151$.

12. J. T. Schwartz, Perturbations of spectral operators and applications. I, Pacific J. Math. vol. 4 (1954) pp. 415-458.

13. E. Schwengeler, Geometrisches über die Verteilung der Nullstellen spezieller ganzer Funktionen (Exponentialsummen), Ph.D. dissertation, Zürich, 1926.

14. M. H. Stone, Irregular differential systems of order two and the related expansion problems, Trans. Amer. Math. Soc. vol. 29 (1927) pp. 23-53.

15. - Linear transformations in Hilbert space and their applications to analysis, Amer. Math. Soc. Colloquium Publications, vol. 15, 1932.

16. L. E. Ward, Some third-order irregular boundary value problems, Trans. Amer. Math. Soc. vol. 29 (1927) pp. 716-745.

New York UNIVERSITY, NEw York, NEw YoRK

Remington Rand Univac Division of Sperry Rand Corporation, NEW YORK, NEW YORK 